\title{
Eventual monotonicity and convergence to travelling fronts for the solutions of parabolic equations in cylinders
}

\author{
by \\ Jean-Michel ROQUEJOFFRE \\ U.F.R. MIG, U.M.R. CNRS 5640, \\ Université Paul Sabatier, \\ 118, route de Narbonne, 31062 Toulouse Cedex, France. \\ E-mail: roque@mip.ups.tlse.fr
}

\begin{abstract}
The paper is concerned with the long-time behaviour of the solutions of a certain class of semilinear parabolic equations in cylinders, which contains as a particular case the multidimensional thermo-diffusive model in combustion theory. We prove, under minimal conditions on the initial values, that the solutions eventually become monotone in the direction of the axis of the cylinder on every compact subset; this implies convergence to travelling fronts. This result is applied to propagation versus extinction problems: given a compactly supported initial datum, sufficient conditions ensuring that the solution will either converge to 0 or to a pair of travelling fronts are given. Additional information on the corresponding equations in finite cylinders is also obtained.
\end{abstract}

RÉsumÉ. - Cet article traite du comportement en grand temps des solutions d'une classe d'équations paraboliques dans des cylindres; ce type d'équation contient le modèle thermo-diffusif multidimensionnel de la théorie de la combustion. Nous montrons que, sous des hypothèses minimales sur la donnée initiale, les solutions deviennent, en temps fini, monotones sur tout compact dans la direction de l'axe du cylindre; ceci implique la convergence vers des ondes progressives. Ce résultat est ensuite appliqué à des problèmes de propagation et d'extinction: une donnée initiale

A.M.S. Classification: 35 B 35, 35 K 57.

Annales de l'Institut Henri Poincaré - Analyse non linéaire - 0294-1449

Vol. 14/97/04/\$ 7.00/

(C) 1997 L'Association Publications de l'Institut Henri Poincaré. Published by Elsevier B.V. All rights reserved 
étant fixée, on donne des conditions suffisantes pour que la solution qui en est issue tende vers 0 , ou converge vers deux fronts se déplaçant dans des directions opposées. On obtient enfin des informations supplémentaires pour des équations dans des cylindres bornés.

\section{INTRODUCTION}

Let $\Sigma$ be the cylinder: $\Sigma=\{(x, y) \in \mathbf{R} \times \omega\}, \omega$ being an open bounded regular subset of $\mathbb{R}^{N-1}$, with $N>1$; the outward normal derivative at $(x, y) \in \mathbb{R} \times \partial \omega$ will be denoted by $\nu(x, y)$, as usual. Consideration is given to the asymptotic behaviour - as $t \rightarrow+\infty$ - of the solutions of the following class of semilinear parabolic equations

$$
u_{t}-\Delta u+\alpha(y) u_{x}=g(y, u) \quad \text { for }(x, y) \in \Sigma,
$$

with Dirichlet or Neumann boundary conditions:

$$
\mathcal{B} u=0 \quad \text { for }(x, y) \in \mathbf{R} \times \partial \omega,
$$

The boundary condition (1.1b) is either Dirichlet or Neumann: $\mathcal{B} u=u$ or $\mathcal{B} u=\frac{\partial u}{\partial \nu}$. Problem (1.1) is complemented by the initial datum

$$
u(0, x, y)=u_{0}(x, y) .
$$

In the whole paper, the functions $\alpha(y)$ and $g(y, u)$ will satisfy

$$
\alpha \in C^{2, \delta}(\bar{\omega}) \text { for some } \delta>0 ; g \in C^{3}(\bar{\omega} \times \mathbb{R})
$$

This is enough to ensure the existence of a local classical solution to (1.1). It will be denoted by $\mathcal{S}(t) u_{0}$ in the sequel, or simply $u(t)$ when no confusion is possible.

We shall make two sorts of assumptions on $g$. The first series of assumptions that we can make is $g(y, u)=g(u)$, with $g(0)=g(1)=0$. This is the natural generalisation of the well-known one-dimensional model

$$
u_{t}-u_{x x}=g(u),
$$

which has received thorough treatment, and is which is now fully understood. The relevant references are stated in [27] and [22]; we will not come back to them. Let us only mention the celebrated reference [11]. In this framework, the function $g$ will fall into one of the following cases. 
Case A1. There exists $\theta \in] 0,1[$ such that

$$
g<0 \text { in }] 0, \theta[\text { and } g>0 \text { in }] \theta, 1[\text {. }
$$

Moreover

$$
g^{\prime}(0)<0, g^{\prime}(1)<0 .
$$

This case will be referred to as the "bistable case"; it often arises in genetics or population dynamics; see [2], [3], [11]. A typical example is the cubic nonlinearity $g(u)=u(1-u)(u-\theta)$.

Case A2. There exists $\theta \in] 0,1[$ such that

$$
g \equiv 0 \text { in }] 0, \theta[\text { and } g>0 \text { in }] \theta, 1[.
$$

Moreover

$$
g^{\prime}(1)<0 .
$$

This case arises in combustion theory, in the framework of the thermodiffusive approximation; see [28] for the physical background; see also [6]. We will sometimes refer to it as the "ignition temperature case".

Case A3. The function $g$ satisfies $g>0$ on $] 0,1[$, and

$$
g^{\prime}(0)>0>g^{\prime}(1)
$$

When $s \mapsto g(s) / s$ is decreasing, this case is often referred to as the Fisher model in population dynamics, or also KPP model. Another interesting subcase, namely the ZFK model [31], arises in combustion theory; in this context $g^{\prime}(0)$ is assumed to be small and $\int_{0}^{1} g(u) d u$ is large. This assumption will be more precisely quantified in Sections 2 and 3; we will refer to it as Case A3/ZFK.

Case B. The second sort of assumption that we can make concerns $y$-dependent nonlinearities. Assume that the solutions of the problem

$$
\begin{aligned}
-\Delta_{y} u & =g(y, u) \quad \text { in } \omega \\
\mathcal{B} u & =0 \quad \text { on } \partial \omega
\end{aligned}
$$

are such that

$(\mathcal{H} 1) \bullet$ there exist two solutions $\psi_{1}(y)<\psi_{2}(y)$ such that $\mu_{1}\left(-\Delta_{y}-\right.$ $\left.g_{u}\left(y, \psi_{i}\right)\right)>0$,

Vol. 14, $\mathrm{n}^{\circ}$ 4-1997. 
$(\mathcal{H} 2) \cdot$ each solution $\psi$ such that $\psi_{1}<\psi<\psi_{2}$ has $\mu_{1}\left(-\Delta_{y}-\right.$ $\left.g_{u}(y, \psi)\right)<0$.

Here, for a second-order elliptic operator $A$ in $\omega$ with boundary operator $\mathcal{B}$, we denote by $\mu_{1}(A)$ its first eigenvalue. We notice that Case $B$ contains Case A1, but not A2-A3. Therefore in the sequel the emphasis will be laid on the two latter cases. We also notice that it is possible to make assumptions on Problem (1.7) that would contain Case A3; see [32]. We will not make them first because we do not wish to make the paper too complicated, second because the physically relevant situation is really Case A $3 /$ ZFK.

Let us once and for all make the operator $\mathcal{B}$ precise. In Case $A$ the natural boundary operator is the Neumann one; we will therefore always take $\mathcal{B} u=\frac{\partial u}{\partial \nu}$.

In Case B, it is more or less equivalent to take Dirichlet and Neumann boundary data, the Dirichlet conditions being more technically delicate to handle. Therefore we will always take $\mathcal{B} u=u$.

Finally, we will also investigate the following system, which is a slight generalisation of Case A2:

$$
\left\{\begin{array}{l}
u_{t}-\Delta u+\alpha(y) u_{x}=v f(u) \\
v_{t}-\Delta v+\alpha(y) v_{x}=-v f(u)
\end{array} \quad \text { for }(x, y) \in \Sigma,\right.
$$

$$
\begin{gathered}
\frac{\partial u}{\partial \nu}=\frac{\partial v}{\partial \nu}=0 \quad \text { for }(x, y) \in \mathbb{R} \times \partial \omega \\
\left\{\begin{array}{ll}
u(t,-\infty, y)=0, & u(t,+\infty, y)=1 \\
v(t,-\infty, y)=1, & v(t,+\infty, y)=0
\end{array} \text { for } y \in \bar{\omega} .\right.
\end{gathered}
$$

The function $f$ will be smooth, and of "ignition temperature" type, i.e.

$$
f \equiv 0 \text { on }[0, \theta], \quad f>0 \text { on }] \theta,+\infty[\text {. }
$$

Physically speaking, in (1.8), $u$ is a temperature and $v$ a mass fraction. When $u_{0}+v_{0} \equiv 1$, we notice that $u+v \equiv 1$, and that (1.8) reduces to (1.1), with $g(u)=(1-u) f(u)$. This system shares a lot of features with case A2, but we will see that the convergence to the waves is not exponential. It will be treated separately.

In each case A1-A3 and B, Problem (1.1.a), (1.1.b) admits travelling front solutions, i.e. solutions of (1.1.a), (1.1.b) of the form $u(t, x, y)=$ 
$\phi(x+c t, y)$. They have first been studied in [6] (case A2), then in [9] (cases A1 and A3). These results have been generalised to $y$-dependent nonlinearities in [32]. The waves are unique in each case except Case A3, where there exists a continuum of speeds $c$, which is bounded from below. In [7] and [26], the waves of Case A2 were found to be asymptotically stable; therefore the question which comes next is whether the solution of (1.1) will converge to a travelling front. We proved in [27] that it was indeed true for cases $\mathrm{A} 1$ and $\mathrm{A} 2$, provided that $u_{0}$ was nondecreasing in $x$. Because of the $x$-translational invariance, we did not see any way to carry over the result for nondecreasing data to more general data.

In [22] we treated case A3 in collaboration with J.F. Mallordy, and classified the long-time behaviour of the solution according to the behaviour at $x=-\infty$ of $u_{0}$. We could basically do so because the wave solutions were stable in weighted spaces where the $x$-translations were not continuous However, for Case A3/ZFK, we did not manage to get rid of the nondecreasing assumption for the minimal speed.

The aim of this paper is to extend the results of [27] for cases AlA3/ZFK and B to non necessarily nondecreasing initial data, and to apply these results to various generalisations, such as the study of propagation versus extinction problems, which are of interest in combustion theory, or to system (1.8). In doing so, we put an end to the problem of the long-time behaviour of solutions of the above class of reaction-diffusion equations. In particular, we generalise to the multidimensional setting the convergence theorems of Fife and McLeod [11] for case A1.

In proving exponential convergence theorems, one usually proceeds in three steps: asymptotic stability, compactness of the orbits, and quasiconvergence. The first step is fully treated in [7] and [26] for case A2; cases A1, A3/ZFK and B are similar, up to technical details. The second step relies, as in [11], on the construction of sub and super solutions having uniform-in-time spatial decay properties.

The ultimate step, namely the existence of a sequence $t_{n} \rightarrow+\infty$ such that

$$
\lim _{n \rightarrow+\infty}\left\|u\left(t_{n}, .\right)-\phi\left(.+c t_{n}, .\right)\right\|_{\infty}=0,
$$

together with the asymptotic stability, implies the exponential convergence. In the case $\alpha \equiv$ Constant it is easy to obtain such a sequence $\left(t_{n}\right)_{n}$ by considering the Liapounov function

$$
V(t)=\int_{\Sigma} e^{-(c+\alpha) x}\left(\frac{1}{2}|\nabla u|^{2}-G(u)\right) d x d y,
$$


or perhaps a slightly modified one in case A1 (see [11]); here $G(u)=$ $\int_{0}^{u} g(v) d v$. The function $V(t)$ is time-decreasing; this, with compactness arguments, yields the sequence $\left(t_{n}\right)_{n}$. In the case $\alpha \neq$ Constant things are unfortunately not so easy; in fact it seems to be impossible to find a suitable Liapounov function. As an alternative solution, one could think of adapting of a deep result of Hirsch [17] about strongly monotone semiflows. Let $X$ be an ordered Banach space, such that the positive cone $X_{+}$has nonempty interior; such a space is called strongly ordered. If a local compact semiflow $\mathcal{S}(t)$ on $X$ is such that

$$
\forall(u, v) \in X^{2}, \quad u<v \Longrightarrow \forall t>0, \mathcal{S}(t) v-\mathcal{S}(t) u \in \operatorname{Int} X_{+},
$$

Hirsch's result states that almost every trajectory is quasi-convergent. This result solves the problem for second-order parabolic equations in bounded domains with Neumann boundary conditions, but does not apply to our case. Matano [23], [24] proves similar results under less stringent hypotheses; in particular he does not need the nonempty interior assumption. However, he assumes the semiflow to be strongly order preserving, i.e., for $u<v$ and $t>0$, there exists $\delta(t)>0$ such that

$$
\forall\left(u^{\prime}, v^{\prime}\right) \in X^{2}, \quad\left\|u^{\prime}-u\right\|_{X},\left\|v^{\prime}-v\right\|_{X} \leq \delta(t) \Longrightarrow \mathcal{S}(t) u^{\prime}<\mathcal{S}(t) v^{\prime} .
$$

As is easily seen, the semiflow generated by a parabolic equation in $\Sigma$ is not strongly order preserving, and to weaken significantly (1.10) seems to be a very difficult task. Therefore our work has the merit of providing a nontrivial example of convergence to equilibrium solutions in a setting to which none of the known theorems applies.

Our approach is different from the above-mentionned ones, and relies on the eventual $x$-monotonicity of the solution on every compact of $\bar{\Sigma}$. This idea that the solution of a parabolic equation will asymptotically bear the same qualitative properties as the elliptic solutions dates back to Jones [19]. He proves, in a weak sense, asymptotic spherical symmetry for initially compactly supported solutions of $u_{t}-\Delta u=g(u)$ in $\mathbb{R}^{N}$, with a bistable $g$. His work, based on reflection-type arguments, is the parabolic analogue of Gidas, Ni, Nirenberg [13]. Our method is not unlike the "sliding method" developped by Berestycki and Nirenberg in [10]; it is in fact its parabolic analogue.

The present work is organised as follows. In Section 2 we collect some results on existence, uniqueness and stability of travelling waves; most of them - but not all - are proved in other papers. In Section 3 we state 
our results precisely; Section 4 is devoted to compactness. The results of Section 3 for Cases A1-A3/ZFK are then proved in Sections 5 to 8 . In Section 7 , multiplicity theorems for the solutions of the steady problem in a finite cylinder are given, and their implications discussed. Section 9 is devoted to Case B, and Section 10 to a system (1.8). Possible further investigations are discussed in Section 11. Finally, additional results on asymptotic stability are proved in Appendix.

\section{EXISTENCE AND ASYMPTOTIC STABILITY OF TRAVELLING FRONTS}

Travelling wave solutions of (1.1.a), (1.1.b) satisfy:

$$
\begin{gathered}
-\Delta \phi+(c+\alpha(y)) \phi_{x}=g(y, \phi) \quad \text { for }(x, y) \in \Sigma, \\
\mathcal{B} u=0 \quad \text { for }(x, y) \in \mathbb{R} \times \partial \omega, \\
\phi(-\infty, y)=\psi_{1}(y), \phi(+\infty, y)=\psi_{2}(y) . \quad \text { for } y \in \bar{\omega}
\end{gathered}
$$

In Cases A1-A3, we will always impose: $\psi_{1} \equiv 0$ and $\psi_{2} \equiv 1$. This is the natural conditions to impose in cases $\mathrm{Al}$ and $\mathrm{A} 3$; in Case $\mathrm{A} 2$ one could take any constant between 0 and $\theta$. In Case $\mathbf{B}$, we recall that $\psi_{1}$ and $\psi_{2}$ are described in the introduction.

There are three paragraphs in this section. The first one sums up known existence, uniqueness and qualitative properties of travelling fronts for cases $\mathrm{A} 1, \mathrm{~A} 2$ and $\mathrm{B}$. The second one is devoted to the precise definition of Case A3/ZFK and to the asymptotic behaviour as $x \rightarrow-\infty$ of the wave of minimal speed. In the third one, precise statement of orbital stability results in the form that will be of use to us are given.

\subsection{Existence, uniqueness and qualitative properties: Cases A1, A2 and $B$}

We collect here existence, uniqueness and asymptotic behaviour - as $|x| \rightarrow+\infty-$ of solutions to (2.1) from the papers of Berestycki, Larrouturou, Lions [6], and Berestycki, Nirenberg [8], [9] in cases A1-A2. For Case B, they are taken from Vega [32].

Let us begin with uniqueness results and qualitative properties. In cases A1-A2 and B, there is at most one solution $(c, \phi)$ to Problem $(2.1)$ in the 
following sense: $c$ is unique and $\phi$ is unique up to a translation of the origin in the $x$-direction. Moreover $\phi$ satisfies: $\phi_{r}>0$. Finally, there exist $\lambda_{+}>0, \lambda_{-}>0$ and two $C^{2}$ functions: $\phi_{-}(y), \phi_{+}(y)$, which are positive on $\bar{\omega}$, such that the following estimates hold:

$$
\begin{aligned}
& \left\|\phi(x, .)-\psi_{1}(y)-e^{\lambda_{-} x} \phi_{--}\right\|_{C^{1, \delta}(\bar{\omega})} \leq C e^{(\lambda \ldots+\varepsilon) x}, \quad \text { in } \Sigma_{-} . \\
& \left\|\phi_{x}(x, .)-\lambda_{-} e^{\lambda_{-\alpha} x} \phi_{-}\right\|_{C^{0, \delta}(\bar{\omega})} \leq C e^{(\lambda \ldots+\xi) x}
\end{aligned}
$$

$$
\begin{aligned}
& \left\|\psi_{2}(y)-\phi(x, .)-e^{-\lambda_{+} x} \phi_{+}\right\|_{C^{1, \delta}(\bar{\omega})} \leq C e^{-\left(\lambda_{+}+\varepsilon\right) x}, \quad \text { in } \Sigma_{+}, \\
& \left\|\phi_{x}(x, .)-\lambda_{+} e^{-\lambda_{+} x} \phi_{+}\right\|_{C^{0, \delta}(\bar{\omega})} \leq C e^{-\left(\lambda_{+}+\varepsilon\right) x}
\end{aligned}
$$

for some $\varepsilon>0$. The function $e^{\lambda_{-} x} \phi_{-}(y)$ is a positive exponential solution of

$$
\begin{aligned}
-\Delta u+(c+\alpha(y)) u_{x}-g_{y}\left(y, \psi_{1}\right) u & =0 \text { in } \Sigma_{-} \\
\mathcal{B} u & =0 \text { on } \mathbb{R}_{-} \times \partial \omega
\end{aligned}
$$

and the function $e^{-\lambda_{+} x} \phi_{+}(y)$ is a positive exponential solution of

$$
\begin{aligned}
-\Delta u+(c+\alpha(y)) u_{x}-g_{y}\left(y, \psi_{2}\right) u & =0 \text { in } \Sigma_{+}, \\
\mathcal{B} u & =0 \text { on } \mathbb{R}_{1} \times \partial \omega
\end{aligned}
$$

A proof of these results may be found in [8].

As for the existence results, they depend on whether case A1, A2 or B holds. In case A1, Berestycki and Nirenberg have proved the existence of a solution $(c, \phi)$ for $(2.1)$. when $\omega$ is convex; a proof of this result may be found in [9]. In case A2, Berestycki, Larrouturou and Lions [6] have shown the existence of a solution to (2.1) for any smooth domain $\omega$. Furthermore, still in case $\mathrm{A} 2$, we have $c+\langle\alpha\rangle\rangle 0$, where $\langle\alpha\rangle$ is the mean value of $\alpha$ in $\omega$. Finally, in case B, Vega [32] showed the existence of a solution $(c, \phi)$ to $(2.1)$. It is to be noticed that Case A1, with $\omega$ convex, implies Assumptions $(\mathcal{H} 1)$ and $(\mathcal{H} 2)$.

Let $y_{0} \in \omega$ be given. In the sequel we will often denote by $\phi$ the unique solution of (2.1) which satisfies:

$$
\phi\left(0, y_{0}\right)=\frac{\psi_{1}\left(y_{0}\right)+\psi_{2}\left(y_{0}\right)}{2} .
$$

Moreover, we will always assume that, when we treat case A, problem (2.1) has solutions; the above statements show that this is true in at least a nontrivial case. 


\subsection{The case A3/ZFK: existence and asymptotic properties of the} waves

In the framework of case $\mathrm{A} 3$, there exists a real number $c_{*}$ such that, for $c \geq c_{*}$, there exists a unique-up to translations-wave solution $\phi_{c}$, which is $x$-increasing in $\bar{\Sigma}$; see [8]. There exists a minimal real number $c$, denoted by $c_{0}$ such that the equation in $\lambda$

$$
\mu_{1}\left(-\Delta_{y}+\lambda(c+\alpha(y))\right)=\lambda^{2}+g^{\prime}(0)
$$

has real solutions; for $c>c_{0}$ there are two positive solutions $\lambda_{-}^{\max }(c)>$ $\lambda_{-}^{\text {min }}(c)$; see [8]. In all cases, we have $c_{*} \geq c_{0}$; the case $\mathrm{A} 3 / \mathrm{ZFK}$ will be defined by $c_{*}>c_{0}$. This is true when $g^{\prime}(0)$ is small and $\int_{0}^{1} g(s) d s$ is large; see [8]. This is precisely the physical assumptions of [33], and we shall see that in this case, the wave $\phi_{c_{*}}$ has common features with the wave of case A2.

For $c>c_{*}$, the wave $\phi_{c}$ satisfies (see [8]) estimates (2.2.b), and estimates (2.2.a) with the exponent $\lambda_{-}^{\min }(c)$. For $c=c_{*}$ something different occurs.

THEOREM 2.1. - The wave $\phi_{c_{*}}$ satisfies (2.2.a), with exponent $\lambda_{-}^{\max }\left(c_{*}\right)$.

Proof. - Assume this is not true, and that (2.2.a) holds with exponent $\lambda_{-}^{m i n}\left(c_{*}\right)$. For small real number $d$, we try to solve

$$
\begin{aligned}
-\Delta u+\left(c_{*}+d+\alpha(y)\right) u_{x} & =g(u) \quad \text { for }(x, y) \in \Sigma, \\
u_{\nu} & =0 \quad \text { for }(x, y) \in \mathbb{R} \times \partial \omega, \\
u(-\infty, y) & =0, u(+\infty, y)=1 \quad \text { for } y \in \bar{\omega}
\end{aligned}
$$

with $u$ close to $\phi_{c}^{*}$. Because $c_{*}>c_{0}$, equation (2.3.a), with $c=c_{*}+d$, and with $|d|<c_{*}-c_{0}$, has two solutions. Let $\phi_{c_{*}}^{-}$correspond to the function $\phi_{-}$in (2.2.a). Let $\phi_{c_{*}+d}^{-}$correspond to (2.5), with $\lambda=\lambda_{-}^{\min }\left(c_{*}+d\right)$, and such that

$$
\lim _{d \rightarrow 0}\left\|\phi_{c_{*}+d}^{-}-\phi_{r_{*}}^{-}\right\|_{C^{1}}=0
$$

Obviously, $\lambda_{-}^{\min }\left(c_{*}+d\right) \rightarrow \lambda_{-}^{\min }\left(c_{*}\right)$ as $d \rightarrow 0$. Let us set, for small $d$ :

$$
e_{d}^{-}(x, y)=\phi_{c_{*}+d}^{-}(y) e^{\lambda_{-}^{m i n}\left(c_{*}+d\right) x}
$$

Let $\Gamma$ be the usual $C^{\infty}$ nonnegative function which takes the value 1 for $x \leq 0$, and 0 for $x>1$. We shall look for $u$ under the form

$$
u(x, y)=\phi_{c_{*}}+\left(e_{d}(x, y)-e_{0}(x, y)\right) \Gamma(x)+w(x, y) .
$$

Vol. $14, n^{\circ} 4-1997$. 
Let us set

$$
r=\min \left(\frac{\lambda_{-}^{\min }\left(c_{*}\right)+\lambda_{-}^{\max }\left(c_{*}\right)}{2}, 2 \lambda_{-}^{\min }(c)\right) .
$$

We shall ask $w(x, y)=O\left(e^{r x}\right)$. The equation for $w$ reads, after a tedious but straightforward calculation:

$$
\begin{gathered}
-\Delta w+\left(c_{*}+d+\alpha(y)\right) \partial_{x} w \\
-\left(g^{\prime}\left(\phi_{c_{*}}+\left(e_{d}-e_{0}\right) \Gamma\right)+f_{d}(x, y, w)\right) w=h_{d}(x, y) \\
\left(1+e^{-r x}\right) w \in L^{\infty}(\Sigma)
\end{gathered}
$$

with

$$
\begin{aligned}
f_{d}(x, y, w)= & \frac{g\left(\phi_{c_{*}}+\left(e_{d}-e_{0}\right) \Gamma+w\right)-g\left(\phi_{c_{*}}+\left(e_{d}-e_{0}\right) \Gamma\right)}{w} \\
& -g^{\prime}\left(\phi_{c_{*}}+\left(e_{d}-e_{0}\right) \Gamma\right) \\
& =O(w)
\end{aligned}
$$

and $\left(1+e^{-r x}\right) h_{d}$ bounded and uniformly continuous in $\bar{\Sigma}$. Let us recall (see [22], Theorem 5.1) that the operator

$$
L=-\Delta+\left(c+\alpha(y) \partial_{x}-g^{\prime}(\phi)\right.
$$

with Neumann boundary conditions, is an isomorphism from its domain to $B_{w_{r}, 0}$-see Definition (2.7) below -. Therefore, for $d$ small enough, the above equation is uniquely solvable in $w$. This contradicts the definition of $c_{*}$.

Remark. - This result has already been proved in the one-dimensional framework in [5], where it seems to appear for the first time.

\subsection{Asymptotic stability and nonautonomous equations}

Everything is now stated in the reference frame of a travelling front, i.e. every wave $\phi$ is now a steady solution. Let $U C(\bar{\Sigma})$ be the space of all bounded, uniformly continuous functions in $\bar{\Sigma}$. For $r \in] 0, \lambda_{-}[$, we set:

$$
w_{r}(x, y)=1+e^{-r x} .
$$

Define the space

$$
B_{u_{r}, 0}=\left\{u \in U C(\bar{\Sigma}): w_{r} u \in U C(\bar{\Sigma})\right\},
$$

equipped with the norm: $\|u\|_{w_{r}, 0}=\left\|w_{r}(x, y) u(x, y)\right\|_{\infty}$. The space $B_{w_{r}, 0}$ is used in [26] to prove a nonlinear stability result for the travelling waves 
of case B, with a suitably chosen real number $r$. In the framework of case Al, the relevant space is $U C(\bar{\Sigma})$.

\section{Notations}

1. Let $Z$ be a Banach space of continuous functions in $\bar{\Sigma}$. For $u \in Z$ and $\delta>0$, the ball of $Z$ with centre $u$ and radius $\delta$ will be denoted by $\mathcal{B}_{Z}(u, \delta)$.

2. For $h \in \mathbb{R}$, the $x$-translation operator will be denoted by $\tau_{h}$, and defined by $\left(\tau_{h} u\right)(x, y)=u(x+h, y)$.

3. Let $A$ be the (unbounded) operator of $X$ with suitable domain $D(A)$ (including the Neumann boundary conditions), given by $A=$ $-\Delta+(c+\alpha(y)) \partial_{x}$.

4. We will denote by $X$ the Banach space $U C(\bar{\Sigma})$ (resp. $B_{w_{\frac{r}{2}}, 0}$ with $r<\frac{\lambda_{-}}{2}$, resp. $\left.\lambda_{-}^{\min }\left(c_{*}\right)<r<\lambda_{-}^{\max }\left(c_{*}\right)\right)$ in cases $\mathrm{Al}$ and $\mathrm{B}$ (resp. case $\mathrm{A} 2$, resp. case $\mathrm{A} 3 / \mathrm{ZFK}$ ). Further, we will denote by $X^{\prime}$ the space $U C(\bar{\Sigma})$ (resp. $B_{w_{r}, 0}$ ) in case $\mathrm{A} 1$ and $\mathrm{B}$ (resp. case A2 and A3/ZFK).

5. Finally, following Sattinger [27], it will sometimes be useful to work with the two Banach spaces, defined for every $\omega>0$ :

$$
\begin{aligned}
& X^{\omega}=\left\{v(t) \in C\left(\mathbb{R}_{+}, X\right): \sup _{t \geq 0} e^{\omega t}\|v(t)\|_{X}<+\infty\right\} \\
& E^{\omega}=\left\{v(t) \in C\left(\mathbb{R}_{+}, \mathbb{R}\right): \sup _{t \geq 0} e^{\omega t}|v(t)|<+\infty\right\}
\end{aligned}
$$

We will need two kinds of results. The first one is a summary of our asymptotic stability results for the wave, and will be used in Sections 4 and 5 . The second one, which is a corollary, is a stability result with respect to small time-dependant perturbations; it will be of use in Section 7.

THEOREM 2.2. - For $v_{0}(x, y) \in X$, let $u(t, x, y)$ be the solution of

$$
u^{\prime}+A u=g(u), \quad u_{0}(0, x, y)=\phi(x, y)+v_{0}(x, y) .
$$

There exist a positive real number $\delta_{0}$, one constant $\omega>0$ and a function $\gamma \in C^{1}\left(\mathcal{B}_{X}\left(0, \delta_{0}\right), \mathbb{R}\right)$ such that, for all $v_{0} \in \mathcal{B}_{X}\left(0, \delta_{0}\right)$, the function $u-\tau_{\gamma\left(v_{0}\right)} \phi$ belongs to $X^{\omega}$.

Theorem 2.2 for case A2 is basically proved in [26]. Its proof for cases $A 1, A 3 / Z F K$ and $B$ is the same as in case $A 2$ in the main lines, but a few details differ in cases $\mathrm{A} 1$ and $\mathrm{B}$.

Corollary 2.3. - For $v_{0}(x, y) \in X$ and $h \in X^{2 \omega}$, let $u(t, x, y)$ be the solution of

$$
u^{\prime}+A u=g(u)+h(t), \quad u_{0}(0, x, y)=\phi(x, y)+v_{0}(x, y)
$$

Vol. $14, n^{\circ} 4-1997$. 
There exist positive real numbers $\delta_{0}, \mu_{0}$, one constant $\omega>0$ and a function $\gamma \in C^{1}\left(\mathcal{B}_{X}\left(0, \delta_{0}\right) \times \mathcal{B}_{X-2 \omega}\left(0, \mu_{0}\right), \mathbb{R}\right)$ such that, for all $v_{0} \in \mathcal{B}_{X}\left(0 . \delta_{0}\right)$ and $h \in \mathcal{B}_{X^{-2 \omega}}\left(0, \mu_{0}\right)$, the function $u-\tau_{\gamma\left(w_{0}, h\right)} \phi$ belongs to $X^{\omega}$.

Both proofs will be sketched in Appendix.

\section{LONG-TIME BEHAVIOUR RESULTS}

First consider Cases A1-A3/ZFK. The initial datum $u_{0}$ will always bc assumed to be between 0 and 1 . For Cases $A 1$ and A2, it will be below $\theta$ for large negative $x$. We will see that, according to how big the set $\left\{u_{0}>\theta\right\}$ is, the solution will either converge to a unique front, or asymptotically vanish, or develop into a pair of two travelling fronts.

\subsection{Convergence to travelling fronts}

The initial datum is assumed to have different limits at $\pm \propto$. In all cases, this implies convergence to a front. In what follows, let us recall that $u(t, x, y)$ is the solution of the Cauchy problem (1.1.a)-(1.1.c).

THEOREM 3.1. - Assume case Al holds and take $u_{0}$ in $U C(\bar{\Sigma})$. Additionally assume that there exist $\theta_{-}<\theta, \theta_{+}>\theta$ such that:

$$
\limsup _{x \rightarrow-\infty} u_{0}(x, y) \leq \theta_{-}, \quad \liminf _{x \rightarrow+\infty} u_{0}(x, y) \geq \theta_{+} .
$$

Then there exist $x_{0} \in \mathbb{R}$ and $\omega>0$ such that

$$
\forall t>0, \quad \sup _{(x, y) \in \bar{\Sigma}}\left|u(t, x, y)-\phi\left(x+x_{0}+c t, y\right)\right|=O\left(e^{-\omega t}\right) .
$$

Theorem 3.1 will be proved at the same time as Theorem 3.7 below about Case B. Turn to case A2 and A3/ZFK. It is now clear why we shall only treat the waves with minimal speed: the wave is stable in spaces where the translation operator is continuous, contrary to the waves with higher specds.

THEOREM 3.2. - Assume case A2 or A3/ZFK holds and take $u_{0}$ in $U C(\bar{\Sigma})$. In case A2, assume that there exists $\theta_{+}>\theta$ and $r>0$ satisfying

$$
\begin{gathered}
u_{0} \in B_{w_{r}, 0}, \quad \liminf _{x \rightarrow+\infty} u_{0}(x, y) \geq \theta_{+}, \quad \text { in Case } A 2 \\
r>\lambda_{-}^{\min }\left(c_{*}\right), \\
u_{0} \in B_{w_{*}, 0}, \quad \liminf _{x \rightarrow+\infty} u_{0}(x, y)>0, \quad \text { in Case } A 3 / Z F K
\end{gathered}
$$

Then the conclusion of Theorem 3.1 is valid. 
We have not only improved the results of [27] by removing the monotonicity assumption, but also by allowing any exponential decay at $-\infty$, which is natural in view of the nonlinear stability assumptions in [26]. In [27], we basically needed $u_{0}$ to remain between two translates of $\phi$ for $x \rightarrow-\infty$.

\subsection{Propagation and extinction results}

Let $(\tilde{c}, \tilde{\phi})$ be the travelling front solution of (1.1.a), (1.1.b) satisfying $\tilde{\phi}(-\infty, y)=1, \tilde{\phi}(+\infty, y)=0$, and (2.4). In case A2, the inequalities $c+\langle\alpha\rangle>0,-\tilde{c}-\langle\alpha\rangle>0$, imply $\tilde{c}<c$. In case A1, we just suppose that this inequality is true; for $N=1$ it holds as soon as the total mass of $g$ is positive. As for Case A3/ZFK, we shall denote by $\tilde{c}_{*}$ the maximal admissible speed. Set $\left.\Sigma_{L}:=\right]-L, L[\times \omega$.

THEOREM 3.3. - Assume case AI holds, and that $\dot{c}<$ c. Take $u_{0} \in U C(\Sigma)$ satisfying the following condition: there exist $\delta>0, \eta>0, L>0$ such that

$$
u_{0} \geq \theta+\eta \text { in } \Sigma_{L}, \quad u_{0} \leq \theta-\eta \text { in } \Sigma \backslash \Sigma_{L+\delta}
$$

\section{Then}

1. If $\delta$ and $L$ are small enough, then $\|u(t)\|_{\infty}=O\left(e^{-\omega t}\right)$ for some $\omega>0$,

2. If $L$ is large enough, then there exist $x_{1}, x_{2} \in \mathbb{B}$ and $\omega>0$ such that

$$
\sup _{(x, y) \in \bar{\Sigma}}\left|u(t, x, y)-\left(\phi\left(x+x_{1}+c t, y\right)+\tilde{\phi}\left(x+x_{2}+\tilde{c} t, y\right)-1\right)\right|=O\left(e^{-\omega t}\right) .
$$

THEOREM 3.4. - Assume case A2 holds. Take $u_{0}$ for which there exist $\delta>0, r>0, \eta>0$ and $L>0$ such that

$u_{0} \geq \theta+\eta$ in $\Sigma_{L}, u_{0} \leq \theta-\eta$ in $\Sigma \backslash \Sigma_{L+\delta},\left|u_{0}(x, y)\right|=O\left(e^{-r|x|}\right)$ as $x \rightarrow \pm \infty$.

Then

1. If $L$ and $\delta$ are small enough, then $\mathcal{S}(t) u_{0}$ goes uniformly to 0 ,

2. If $L$ is large enough, then there exist $x_{1}, x_{2} \in \mathbb{R}$ and $\omega>0$ such that

$$
\sup _{(x, y) \in \bar{\Sigma}}\left|u(t, x, y)-\left(\phi\left(x+x_{1}+c t, y\right)+\tilde{\phi}\left(x+x_{2}+\tilde{c} t, y\right)-1\right)\right|=O\left(e^{-\omega t}\right) .
$$

Turn to Case A3/ZFK. We only consider initial data with compact supports; the generalisation to data having prescribed exponential decays at $\pm \infty$ can be made by the interested reader with the aid of [22]. 
THEOREM 3.5. - Assume case A3/ZFK holds. Take $u_{0} \in U C(\bar{\Sigma})$ with nonempty compact support. There exist $x_{1}, x_{2} \in \mathbb{R}$ and $\omega>0$ such that

$$
\sup _{(x, y) \in \bar{\Sigma}}\left|u(t, x, y)-\left(\phi_{c_{*}}\left(x+x_{1}+c_{*} t, y\right)+\tilde{\phi}_{\tilde{c}_{*}}\left(x+x_{2}+\tilde{c}_{*} t, y\right)-1\right)\right|=O\left(e^{-\omega t}\right) .
$$

Theorems 3.3 and 3.4 are the multidimensional analogues of Theorem 3.2 in [11] and Theorem 3.2 in [2]. Theorem 3.5 seems to appear for the first time, even in the $1 \mathrm{D}$ case. In this latter case, the reader may see that, if $u_{0}$ does not have a compact support but decays exponentially at one or two of the ends of the cylinder, one may have many combinations of diverging fronts. These three results will be proved in Sections 6 and 7.

The first four theorems admit a corollary, which describes the long-time behaviour of the solution when $u_{0}$ is assumed to be above $\theta$ only in cylinders of the form $\mathbb{R} \times \omega^{\prime}$, with $\omega^{\prime} \subset \omega$, and this is the purpose of Theorem 3.6 below. We will restrict ourselves to Case A2, because the applications that we really have in mind are related to the thermo-diffusive model for flame propagation. The interested reader may state analogous results for the bistable case.

For $\omega^{\prime} \subset \omega, \omega^{\prime}$ measurable, we set $\left.\Sigma_{L, \omega^{\prime}}=\right]-L, L\left[\times \omega^{\prime}\right.$ and $\Sigma_{\omega^{\prime}}=\mathbb{R} \times \omega^{\prime}$.

THEOREM 3.6. - Assume case A2 holds, and take $u_{0} \in U C(\bar{\Sigma})$, compactly supported.

1. Assume there exist $\omega^{\prime \prime} \subset \omega^{\prime} \subset \omega$ and $\left.\eta \in\right] 0, \theta[$ such that

$$
u_{0} \geq \theta+\eta \text { in } \Sigma_{\omega^{\prime \prime}}, \quad u_{0} \leq \theta-\eta \text { in } \Sigma_{\omega \backslash \bar{\omega}^{\prime}}
$$

If $\left|\omega^{\prime \prime}\right|$ and $\left|\omega^{\prime} \backslash \omega^{\prime \prime}\right|$ are small enough, then $\mathcal{S}(t) u_{0}$ drops uniformly below $\theta$ in finite time. Conversely, if $\left|\omega \backslash \omega^{\prime \prime}\right|$ is small enough, then the conclusion of Theorem 3.2 holds.

2. Assume there exist $\omega^{\prime \prime} \subset \omega^{\prime} \subset \omega, \delta>0, r>0, \eta>0$ and $L>0$ such that

$$
\begin{gathered}
u_{0} \geq \theta+\eta \text { in } \Sigma_{L, \omega^{\prime \prime}}, u_{0} \leq \theta-\eta \text { in } \Sigma \backslash \Sigma_{L+\delta, \omega^{\prime}}, \\
\left|u_{0}(x, y)\right|=O\left(e^{-r|x|}\right) \text { as } x \rightarrow \pm \infty .
\end{gathered}
$$

If $L$ is large enough and $\left|\omega \backslash \omega^{\prime \prime}\right|$ is small enough, then the conclusion of Theorem 3.4 holds.

Theorem 3.6 has a very practical meaning: if one believes in the accuracy of the thermo-diffusive model, one sees that a tlame has to be initiated in a large part of the cylinder, if one wishes it to propagate. 
Theorem 3.6 is proved in Sections 6-7, as a corollary of Theorems 3.2 and 3.4 .

\subsection{Generalisations}

First, let us consider case B. A reasonable assumption on $u_{0}$ is to ask $\psi_{1} \leq u_{0} \leq \psi_{2}$. To formulate a slightly more general assumption, we introduce the problem

$$
\begin{aligned}
\psi_{t}-\Delta \psi & =g(y, \psi) \quad \text { in } \omega \\
\psi & =0 \quad \text { on } \partial \omega
\end{aligned}
$$

Let $\rho_{0}>0$ such that, if $\left\|\psi_{0}-\psi_{1}\right\|_{\infty}<\rho_{0}$ (resp. $\left\|\psi_{0}-\psi_{2}\right\|_{\infty}<\rho_{0}$ ), then the solution of (3.6) starting from $\psi_{0}$ is attracted by $\psi_{1}$ (resp. $\psi_{2}$ ). Let us set, for $u_{0} \in U C(\bar{\Sigma})$ :

$$
\begin{gathered}
\eta_{1}\left(u_{0}\right)=\max \left(\limsup _{x \rightarrow-\infty} \sup _{y \in \omega}\left|u_{0}(x, y)-\psi_{1}(y)\right|,\right. \\
\left.\limsup _{x \rightarrow+\infty} \sup _{y \in \omega}\left|u_{0}(x, y)-\psi_{2}(y)\right|\right) \\
\eta_{2}\left(u_{0}\right)=\max \left(\|\| u_{0}\left\|_{\infty}-\psi_{1}\right\|_{\infty},\|\| u_{0}\left\|_{\infty}-\psi_{2}\right\|_{\infty}\right)
\end{gathered}
$$

THEOREM 3.7. - Assume Case B holds, and assume that $\max \left(\eta_{1}\left(u_{0}\right), \eta_{2}\left(u_{0}\right)\right) \leq \rho_{0}$. Then the conclusion of Theorem 3.1 holds.

It finally remains to deal with System (1.8).

THEOREM 3.8. - Assume that the initial datum $\left(u_{0}, v_{0}\right)$, together with satisfying (1.8.c), is such that $u_{0}$ and $1-v_{0}$ belong to $B_{w_{r}, 0}$ for some $r>0$. Then there exists $x_{0} \in \mathbb{R}$ such that

$$
\begin{gathered}
\lim _{t \rightarrow+\infty} \sup _{(x, y) \in \bar{\Sigma}}\left(\left|u(t, x, y)-\phi\left(x+x_{0}+c t, y\right)\right|\right. \\
+\left|v(t, x, y)-1+\phi\left(x+x_{0}+c t, y\right)\right|=0 .
\end{gathered}
$$

Theorems 3.7 and 3.8 are closely related to Theorems 3.1 and 3.2. They will be treated in separate sections, with the emphasis laid on the additional difficulties.

\section{COMPACTNESS IN $X$}

In [11], Fife and McLeod get compactness by confining $\mathcal{S}(t) u_{0}$ between two functions $\underline{u}$ and $\bar{u}$, each of them converging to a travelling front. In this scope, they constructed two functions $q_{i}(t) \rightarrow 0$ and $\xi_{i}(t) \rightarrow \xi_{i}^{\infty} \in \mathbb{R}$, such that $\underline{u}(t)=\phi\left(x+c t+\xi_{1}(t)\right)-q_{1}(t)\left(\right.$ resp. $\left.\bar{u}(t)=\phi\left(x+c t+\xi_{2}(t)\right)-q_{2}(t)\right)$ is a subsolution (resp. a supersolution).

Vol. 14, $n^{\circ}$ 4-1997. 
Their construction can be very easily extended to the multi-D setting in case A1, and we did so in [27]; however we could not carry it over to case A2 with the natural assumption. We give here an alternative construction, based on Theorem 2.2. It will be convenient to work in the reference frame of a travelling wave; we still denote by $x$ and $y$ the new coordinates.

LEMMA 4.1.

1. (Case $A l$ and $B$ ). Let $u_{0}$ be as in Theorem 3.1 or 3.7. There exist $q>0$, $\omega>0$, and $\xi_{1}<\xi_{2}$ such that, for $t_{0}$ large enough, there holds:

$$
\forall t \geq t_{0}, \quad \tau_{\xi_{1}} \phi-q e^{-\omega t} \leq \mathcal{S}(t) u_{0} \leq \tau_{\xi_{2}} \phi+q e^{-\omega t}
$$

2. (Case $A 2$ and $A 3 / Z F K$ ). Let $u_{0}$ be as in Theorem 3.2. For $t_{0}>0$ large enough there exist $q>0, \omega>0$, and $\xi_{1}<\xi_{2}$ such that:

$$
\forall t \geq t_{0}, \quad \tau_{\xi_{1}}\left(\phi-q / w_{\frac{r}{2}} e^{-\omega t}\right) \leq \mathcal{S}(t) u_{0} \leq \tau_{\xi_{2}}\left(\phi+q / w_{\frac{r}{2}} e^{-\omega t}\right)
$$

The proof relies on Theorem 2.1. The following lemma will be useful.

LEMMA 4.2.

1. Assume case Al or $B$ holds, and let $u_{0}$ fall in the relevant assumptions. Let $\eta_{i}$ be defined as in (3.7). Then

$$
\lim _{t \rightarrow+\infty}\left(\eta_{1}\left(\mathcal{S}(t) u_{0}\right)+\eta_{2}\left(\mathcal{S}(t) u_{0}\right)\right)=0
$$

2. Assume case $A 2$ or A3/ZFK holds, and that $u_{0}$ is as in Theorem 3.2 . Then, for all $t \geq 0, u(t)$ belongs to $B_{w_{r}, 0}$; moreover it satisfies

$$
\lim _{t \rightarrow+\infty}\left(\liminf _{x \rightarrow+\infty} u(t, x, y)\right)=1 .
$$

Proof. - As for $\eta_{1}$, the proof is an easy adaptation of Lemmas 2.2 and 2.3 in [27]; therefore it will be omitted. As for $\eta_{2}$, it is hardly more complicated: we simply have

$$
\mathcal{S}(t)\left(\psi_{1}+\eta_{2}\left(u_{0}\right)\right) \leq \mathcal{S}(t) u_{0} \leq \mathcal{S}(t)\left(\psi_{1}+\eta_{2}\left(u_{0}\right)\right)
$$

because $\eta_{i}\left(u_{0}\right) \leq \rho_{0}$ we have $\mathcal{S}(t)\left(\psi_{i}-\eta_{2}\left(u_{0}\right)\right) \rightarrow \psi_{i}$.

Proof of Lemma 4.1.

1. Bistable case. Let $\phi$ be a travelling wave. Set $v_{0} \equiv 1$, and notice that $v_{0} \in X$; let $\delta_{0}>0$ be as in Theorem 2.1 and set $\varepsilon=\frac{\delta_{0}}{2}$. From Lemma 4.2 , part 1 , select $t_{0}>0$ large enough so that

$$
\eta_{1}\left(\mathcal{S}\left(t_{0}\right) u_{0}(x, y)\right)+\eta_{2}\left(\mathcal{S}\left(t_{0}\right) u_{0}(x, y)\right) \leq \frac{\varepsilon}{2}
$$


Therefore there exists $M>0$ such that

$$
\tau_{-M}\left(\phi-\varepsilon v_{0}\right) \leq \mathcal{S}\left(t_{0}\right) u_{0} \leq \tau_{M}\left(\phi+\varepsilon v_{0}\right) .
$$

From the maximum principle, we have, for all $t \geq t_{0}$ :

$$
\mathcal{S}\left(t-t_{0}\right)\left(\tau_{-M}\left(\phi-\varepsilon v_{0}\right)\right) \leq \mathcal{S}(t) u_{0} \leq \mathcal{S}\left(t-t_{0}\right)\left(\tau_{M}\left(\phi+\varepsilon v_{0}\right)\right) .
$$

The equation and boundary conditions being $x$-independant, the semigroup $\mathcal{S}(t)$ commutes with the $x$-translation operator; we get therefore

$$
\tau_{-M}\left(\mathcal{S}\left(t-t_{0}\right)\left(\phi-\varepsilon v_{0}\right)\right) \leq \mathcal{S}(t) u_{0} \leq \tau_{M}\left(\mathcal{S}\left(t-t_{0}\right)\left(\phi+\varepsilon v_{0}\right)\right)
$$

Application of Theorem 2.1 to $\phi \pm \varepsilon v_{0}$ and use of the continuity in $X$ of the $x$-translation operator exactly yields (4.1), with $x_{1}=-M-\varepsilon \gamma(-\varepsilon)$, $x_{2}=M+\varepsilon \gamma(\varepsilon)$ and some $q>0$.

2. Ignition temperature case. It should be noticed that, in Theorem 3.2, $r$ may be assumed to be so small that Theorem 2.1 holds in $B_{w_{r}, 0}$. This will be understood to hold without further notice.

Let $\phi$ be a travelling wave. Set $v_{0}=1 / w_{\frac{r}{2}}$, and select $\varepsilon_{0}>0$ so small that Theorem 2.1 holds with $\varepsilon$ and $v_{0}$. Choose $t_{0}$ large enough so that, by virtue of Lemma 4.2, part 2, one has:

$$
\liminf _{x \rightarrow+\infty} u\left(t_{0}, x, y\right) \geq 1-\frac{\varepsilon}{2},
$$

and remember that $\mathcal{S}\left(t_{0}\right) u_{0}$ still lies in $B_{w_{r}, 0}$. Therefore one may find $M>0$ large enough so that

$$
\tau_{-M}\left(\phi-\varepsilon v_{0}\right) \leq \mathcal{S}\left(t_{0}\right) u_{0} \leq \tau_{M}\left(\phi+\varepsilon v_{0}\right) .
$$

Arguing as in part 1 of this lemma yields (4.2).

3. ZFK case. Same treatment as the ignition temperature case.

Corollary 4.3. - Select $\delta>0$. In cases AI-A3/ZFK and B, the set $\left\{\mathcal{S}(t) u_{0}, t \geq \delta\right\}$ is relatively compact in $X$.

The proof relies on Lemma 4.1 and Ascoli's Theorem; it is by now standard and we omit it. The reader may refer to [11].

Armed with Lemmas 4.1 and 4.3 , we may start the proof of the first three convergence theorems. 


\section{QUASICONVERGENCE AND PROOFS OF THEOREMS 3.1-3.2}

This section presents the basic version of our results, namely Theorems 3.1 and 3.2. The cornerstone of our argument is Proposition 5.2 below, which states that any $\omega$-limit contains at least one $x$-increasing element. The section is divided into three paragraphs: in the first one, we state a boundary form of the Harnack inequalities; in the second one, we state and prove Proposition 5.2. Finally, we end the proofs of Theorems 3.1 and 3.2. As in Section 4, we work in the reference frame of a travelling front.

\subsection{A boundary form of the parabolic Harnack inequalities}

On $Q=\mathbb{R}_{+} \times \Sigma$ consider a nonnegative strong solution of

$$
u_{t}-\Delta u+B(t, x, y) \cdot \nabla u+c(t, x, y) u=0
$$

with Neumann boundary conditions. The coefficients $B$ and $c$ belong to $C^{\delta, \frac{\delta}{2}}(\bar{Q})$; moreover we assume

$$
\|B\|_{L^{\infty}(Q)}+\|c\|_{L^{\infty}(Q)} \leq \mu
$$

From the usual Harnack inequalities [18] one can prove

Proposition 5.1. - For every $M>0$, there exists $T(M)>0$, $\delta T(M)<T(M)$ and $K(M, \mu) \geq 0$ such that:

$$
\forall t \in] T(M),+\infty[
$$

$$
\inf _{\substack{t \geq s \geq t-\delta T(M) \\(x, y) \in \bar{\Sigma}_{M}}} u(s, x, y) \geq K(M, \mu) \sup _{\substack{t-T(M)+\delta T(M) \geq s \geq t-T(M) \\(x, y) \in \bar{\Sigma}_{M}}} u(s, x, y) .
$$

The elliptic version of this result may be found in [4]. We have stated the form that will be of use to us. Proof and comments can be found in [27].

\subsection{A property of the $\omega$-limit sets}

Recall that the $\omega$-limit set of $u_{0} \in X$ with respect to the semiflow $\mathcal{S}(t)$ is the set

$$
\omega\left(u_{0}\right):=\left\{\psi \in X: \exists\left(t_{n}\right)_{n} / \lim _{n \rightarrow+\infty} t_{n}=+\infty \text { and } \lim _{n \rightarrow+\infty} \mathcal{S}\left(t_{n}\right) u_{0}=\psi\right\} .
$$

In both cases $\mathbf{A}$ and $\mathbf{B}$, any $\omega$-limit set is nonempty and compact by Corollary 4.3; moreover, as is well-known, it is connected and positively invariant. We want to prove the following 
Proposition 5.2. - For every $u_{0} \in X$, there exists $\psi \in \omega\left(u_{0}\right)$ which is $x$-increasing.

We will see that this property forces the solution to become $x$-increasing on every compact subset of $\bar{\Sigma}$ in finite time. Notice that, due to the standard parabolic estimates [20], the function $\psi$ belongs to $W^{3, \infty}(\Sigma)$. Furthermore, we may suppose that $\psi_{x}>0$ in $\bar{\Sigma}$. Indeed, if it were not true, we would still have $(\mathcal{S}(1) \psi)_{x}>0$ in $\bar{\Sigma}$, due to the strong maximum principle.

Let $u_{0} \in X$ be selected once and for all. From Lemma 4.1, there exist two real numbers $h_{1} \leq h_{2}$ such that

$$
\forall \psi \in \omega\left(u_{0}\right), \quad \tau_{h_{1}} \phi \leq \psi \leq \tau_{h_{2}} \phi .
$$

Denote by $h$ the following application, defined on $\omega\left(u_{0}\right)$ :

$$
\forall \psi \in \omega\left(u_{0}\right), \quad h(\psi)=\operatorname{Inf}\left\{h>0: \forall k \geq h, \tau_{k} \psi \geq \psi\right\} ;
$$

from (5.4) we see that $h(\psi)$ is finite for $\psi \in \omega\left(u_{0}\right)$. Also, as can easily be noticed, the function $h$ is nonnegative lower semicontinuous on $\omega\left(u_{0}\right)$; therefore, by compactness, it attains its minimum $h_{0}$ at some point $\psi_{0} \in \omega\left(u_{0}\right)$. We will prove by contradiction that $h_{0}=0$.

Assume therefore that $h_{0}>0$. Notice that, from the definition of $h_{0}$ and the maximum principle, we have $h\left(\mathcal{S}(t) \psi_{0}\right)=h_{0}$. The contradiction will follow from two intermediate lemmas.

Lemma 5.3. - There exists $\mu>0$ such that

$$
\forall t \geq 0, \quad \sup _{(x, y) \in \bar{\Sigma}}\left(\mathcal{S}(t) \psi_{0}\left(x+h_{0}, y\right)-\mathcal{S}(t) \psi_{0}(x, y)\right) \geq \mu .
$$

Proof. - If this were not true, there would exist a sequence $\left(t_{n}\right)_{n}$ such that:

$$
\begin{aligned}
& \text { (i) } \lim _{n \rightarrow+\infty} t_{n}=t_{\infty} \in \overline{\mathbf{R}}, \\
& \text { (ii) } \lim _{n \rightarrow+\infty} \mathcal{S}\left(t_{n}\right) \psi_{0}=\psi_{\infty} \in \omega\left(u_{0}\right), \\
& \text { (iii) } \tau_{h_{0}} \psi_{\infty}=\psi_{\infty} .
\end{aligned}
$$

However, whether $t_{\infty}$ belongs to $\mathbb{R}$ or not, (iii) cannot be true because of (5.4). Hence Lemma 5.3 holds.•

For every $\delta \in \mathbb{R}$, let $v^{\delta}(t, x, y)$ be defined as

$$
v^{\delta}(t, x, y)=\mathcal{S}(t) \psi_{0}\left(x+h_{0}-\delta, y\right)-\mathcal{S}(t) \psi_{0}(x, y)
$$

We will turn estimate (5.5) into a lower estimate for $v^{\delta}$ on every compact. Vol. $14, n^{\circ} 4-1997$. 
LEMma 5.4. - For every $M>0$ large enough, there exist $\left.\delta_{0}(M) \in\right] 0, h_{0}[$ and $\mu(M)>0$ such that

$$
\forall \delta \in\left[0, \delta_{0}(M)\left[, \forall t \geq 1, \forall(x, y) \in \Sigma_{M}, \quad v^{\delta}(t, x, y) \geq \mu(M)\right.\right.
$$

Proof. - Let $t_{0} \geq 1$ be as in Lemma 4.1; also assume that it is large enough so that, for all $M>0$ large enough and for all $t \geq t_{0}$, the left handside of inequality (5.5) is attained on $\bar{\Sigma}_{M}$. The function $v^{0}$ is a solution of

$$
\begin{aligned}
v_{t}^{0}+A v^{0}+\frac{g\left(\tau_{h_{0}} u\right)-g(u)}{\tau_{h_{0}} u-u} v^{0} & =0 \quad \text { in } \Sigma, \\
\frac{\partial v^{0}}{\partial \nu} & =0 \quad \text { on } \mathbb{R} \times \partial \omega ;
\end{aligned}
$$

notice that the coefficients of the equation satisfy assumptions (5.2) and that $v^{0}$ is nonnegative. From Lemma 5.3, the quantity $\sup _{(x, y) \in \Sigma_{M}} v^{0}(t, x, y)$ is estimated from below on $\left[t_{0},+\infty[\right.$ by a positive constant; by the strong maximum principle we may in fact write

$$
\inf _{t \geq 1}\left(\sup _{(x, y) \in \Sigma_{M}} v^{0}(t, x, y)\right) \geq 2 \mu(M)
$$

for some $\mu(M)>0$. But we have $\sup _{(t, x, y) \in \mathbb{P} \times \bar{\Sigma}} u_{x}(t, x, y)<+\infty$ from the standard parabolic estimates; hence the existence of $\delta_{0}(M)$ is guaranteed.

Proof of Proposition 5.2. - Let $\theta_{1}$ be defined as follows:

1. There exists $\gamma>0$ such that $g^{\prime} \leq-\gamma$ on $\left[1-\theta_{1}, 1\right]$,

2. If case A1 holds, there exists $\gamma>0$ such that $g^{\prime} \leq-\gamma$ on $\left[0, \theta_{1}\right]$; if case $\mathrm{A} 2$ holds then $\theta_{1} \leq \frac{\theta}{2}$.

3. If case A3/ZFK holds, then there exists $\gamma>0$ such that $g^{\prime}<\gamma$ on $\left[0, \theta_{1}\right]$, and Problem (2.5), with $\gamma$ instead of $g^{\prime}(0)$, has two real positive solutions

From (5.4), there exists $M>0$ such that

$$
\left.\left.\forall(t, x, y) \in \mathbb{R}_{+}\right]-\infty,-M\right] \times \omega, \quad 0 \leq \mathcal{S}(t) \psi_{0}(x+k, y) \leq \theta_{1} ;
$$

$$
\forall k \in\left[0, h_{0}\right],
$$

$$
\forall(t, x, y) \in \mathbb{R}_{+}\left[M,+\infty\left[\times \omega, \quad 1-\theta_{1} \leq \mathcal{S}(t) \psi_{0}(x+k, y) \leq 1 .\right.\right.
$$

If case $\mathrm{A} 1$ or $\mathrm{A} 2$ holds, we take $\left.\theta_{1} \in\right] 0, \inf \left(\theta, 1-\theta, \frac{1}{2}\right)[$. Let $M>0$ be chosen once and for all such that both Lemma 5.4 and (5.7) hold for $M$. 
We are now going to study the evolution of $\mathcal{S}(t) \psi_{0}$. By compactness, there exist a sequence $\left(t_{n}\right)_{n}$ and $\psi_{\infty} \in \omega\left(u_{0}\right)$ such that

$$
\lim _{n \rightarrow+\infty}\left\|\mathcal{S}\left(t_{n}\right) \psi_{0}-\psi_{\infty}\right\|_{x}=0
$$

From the definition of $h_{0}$ and Lemma 5.4, we know that, for all $(x, y) \in \Sigma_{M}$ and $k \geq h_{0}-\delta_{0}, \psi_{\infty}(x+k, y) \geq \psi_{\infty}(x, y)$. Let us see what happens for $x \geq M$. From Point 1 of the definition of $\theta_{1}$, there exists a continuous function $p(t, x, y) \leq-\gamma$ such that, or all $\delta \in\left[0, \delta_{0}\right]$, the function $v^{\delta}$ satisfics

$$
\begin{aligned}
& \left.v_{t}^{\delta}+A v^{\delta}-p(t, x, y) v^{\delta}=0 \text { for }(x, y) \in\right] M,+\infty[\times \omega \\
& \left.\frac{\partial v^{\delta}}{\partial \nu}=0 \text { for }(x, y) \in\right] M,+\infty\left[\times \partial \omega, \quad v^{\delta}(t, M, y) \geq 0 .\right.
\end{aligned}
$$

Therefore, by the maximum principle, there exists $q>0$ such that, for all $(x, y) \in\left[M,+\infty\left[\times \omega\right.\right.$, the inequality: $v^{\delta}(t, x, y) \geq-q e^{-\gamma t}$ holds. This implies that $\psi_{\infty}(x+k, y) \geq \psi_{\infty}(x, y)$ for $(x, y) \in[M,+\infty[\times \omega$ and $k \geq h_{0}-\delta_{0}$.

To see what happens for $x \leq-M$, we have to distinguish between each case A1-A3. The same argument as above works for case A1, therefore turn to case A2. This is not much more complicated, because $v^{\delta}$ is larger than a negative solution of te pure convection-diffusion equation in the half cylinder, with a zero limit at $x=-\infty$. Lemma 6.1 below asserts that such a solution tends to zero as $t \rightarrow+\infty$.

It therefore remains to treat Case A3/ZFK. We follow an idea of [26].

Set $\beta_{*}=c_{*}+\langle\alpha\rangle$; recall that $\beta_{*}>0$. For any $\left.\eta \in\right] 0, \beta_{*}[$ let $\rho(\eta)$ be the principal generalized eigenvalue for the problem:

$$
\begin{gathered}
-\Delta_{y} \psi+r(\alpha(y)-<\alpha>+\eta) \psi=\left(r^{2}+\gamma\right) \psi \quad \text { in } \omega \\
\frac{\partial \psi}{\partial \nu}=0 \quad \text { on } \partial \omega .
\end{gathered}
$$

Let $\eta$ be chosen once and for all so small that $0<\rho(\eta)<$ $\frac{\lambda_{-}^{\max }\left(c_{*}\right)+\lambda_{-}^{\min }\left(c_{*}\right)}{2}$. The existence of $\rho(\eta)$, follows from point 3 and [8], Section 3 . We denote by $\rho$ the real number $\rho(\eta)$, and by $\psi_{-}(y)$ one of the corresponding positive eigenfunctions. The function $\tilde{v}^{\delta}(t, x, y)$, defined by

$$
v^{\delta}(t, x, y)=e^{\rho x} \psi_{-}(y) \tilde{v}^{\delta}(t, x, y)
$$

Vol. 14, $n^{\circ} 4-1997$ 
satisfies the equation

$$
\begin{aligned}
& \tilde{v}_{t}^{\delta}-\Delta \tilde{v}^{\delta}-2 \frac{\nabla_{y} \psi_{-}}{\psi_{-}} . \nabla_{y} \tilde{v}^{\delta}+ \\
&\left(c_{*}+\alpha(y)-2 \rho\right) \frac{\partial \tilde{v}^{\delta}}{\partial x} \\
&\left.+\left(\rho \beta_{*}+\tilde{p}(t, x, y)\right) \tilde{v}^{\delta}=0 \text { in }\right]-\infty,-M[\times \omega, \\
&\left.\frac{\partial \tilde{v}^{\delta}}{\partial \nu}=0 \text { for }(x, y) \in\right]-\infty,-M[\times \partial \omega \\
& \tilde{v}^{\delta}(t,-M, y) \geq 0 \quad \tilde{v}^{\delta}(t,-\infty, y)=0 .
\end{aligned}
$$

The coefficient $\tilde{p}$ is nonnegative. Therefore, there exists $q>0$ such that, for all $(x, y) \in]-\infty,-M\left[\times \omega\right.$, the inequality: $v^{\delta}(t, x, y) \geq-q e^{-\rho \beta_{*} t}$ holds.

To sum up, we have just constructed, in all the three cases, $\psi_{\infty} \in \omega\left(u_{0}\right)$ such that, for every $k \geq h_{0}-\delta_{0}$, the inequality $\tau_{k} \psi_{\infty} \geq \psi_{\infty}$ holds, which implies: $h\left(\psi_{\infty}\right) \leq h_{0}-\delta_{0}$. This contradicts the definition of $h_{0}$.

\subsection{Eventual monotonicity and convergence}

We are now able to prove, in a few lines, Theorems 3.1 and 3.2. However, a consequence of Proposition 5.2 is finite time monotonicity on every compact set. We are going to dwell on this property for a while, because this is really the effect that allows convergence.

Proposition 5.5. - For every $M>0$, there exists $T(M)>0$ such that, for all $t \geq T(M)$, we have: $\left(\mathcal{S}(t) u_{0}\right)_{x}>0$ on $\Sigma_{M}$.

Proof. - Let $\psi \in \omega\left(u_{0}\right)$ be such that $\psi_{x}>0$ in $\bar{\Sigma}$, and let $\left(t_{n}\right)_{n}$ be such that $\left\|\mathcal{S}\left(t_{n}\right) u_{0}-\psi\right\|_{X}$ and $\left\|\left(\mathcal{S}\left(t_{n}\right) u_{0}\right)_{x}-\psi_{x}\right\|_{X}$ go to 0 .

A consequence of Propositions 4.4 and 5.3 of [27] is the fact that every $x$-nondecreasing orbit is $L^{\infty}$-stable; in particular there exist $C(\psi)>0$ and $\varepsilon_{0}(\psi)>0$ such that, for $\varepsilon \leq \varepsilon_{0}(\psi)$, and for all $u_{0} \in \mathcal{B}_{X^{\prime}}(\psi, \varepsilon)$, the following inequality holds:

$$
\forall t \geq 0,\left\|\mathcal{S}(t) \psi-\mathcal{S}(t) u_{0}\right\|_{X}+\left\|(\mathcal{S}(t) \psi)_{x}-\left(\mathcal{S}(t) u_{0}\right)_{x}\right\|_{x} \leq C(\psi) \varepsilon
$$

Further, from Proposition 5.1 and (5.4), for every $M>0$, there exists $\mu(M)>0$ such that $(\mathcal{S}(t) \psi)_{x} \geq \mu(M)$ for every $t \geq t_{0}$ on $\Sigma_{M}$. This proves Proposition 5.5.

We could appeal to Proposition 5.5 to end the proof of our convergence theorems. However, in [27], we obtained convergence results for $x$ nondecreasing orbits; so we are going to use them. 
Proof of Theorem 3.1 and 3.2. - Theorems 1.1 and 1.2 of [27] assert the following: when $u_{0}$, besides satisfying assumptions (3.1) (bistable case) or (3.2) (ignition temperature case), is $x$-nondecreasing, then $\mathcal{S}(t) u_{0}$ converges to a travelling front. As for Case A3/ZFK, we use Theorem 12 of [22]. In fact, in the ignition temperature case, Theorem 1.2 is stated when $\frac{u_{0}}{\phi}$ is between two constants, but it works perfectly with assumptions (3.2): what we in fact needed was Lemma 4.1.

Let once again $\psi \in \omega\left(u_{0}\right)$ be such that $\psi_{x}>0$ in $\bar{\Sigma}$. From (5.4), it perfectly fulfils the assumptions of Theorems 1.1 and 1.2 of [27]. From these theorems, we infer that $\mathcal{S}(t) \psi$ converges to $\tau_{h} \phi$, for some real number $h$. From the closedness of $\omega\left(u_{0}\right), \tau_{h} \phi \in \omega\left(u_{0}\right)$. This is exactly quasiconvergence. Now, as we said in introduction, this property, together with asymptotic stability, implies the convergence of $\mathcal{S}(t) u_{0}$ towards $\tau_{h} \phi \bullet \bullet$

\section{CONVECTION-DIFFUSION EQUATION, EXTINCTION, REFINED CONDITIONS FOR THE CREATION OF ONE FRONT}

In [2] and [3], Aronson and Weinberger noticed that exctinction occurs when the part of $\Sigma$ in which the initial datum is above $\theta$ is small enough; their analysis required two ingredients. The first one is the time-decay property of the linear heat equation; the second is the possibility of converting an initial $L^{2}$-estimate into a not much bigger $L^{\infty}$ estimate for later time. All that we will have to do is really to check these two properties, and this section is nothing but rewriting in terms of our nonselfadjoint problem the estimates of [2] and [3] for the particular case of the heat equation. In what follows, $\Delta_{y}$ will always be understood with Neumann boundary conditions.

LEMMA 6.1. - Let $p>N$ be chosen.

1. There exists $C_{p}>0$ such that, for all $W_{0} \in L^{p}(\Sigma)$ :

$$
\forall t>0, \quad\left\|e^{-t A} W_{0}\right\|_{L^{\infty}(\Sigma)} \leq C_{p}\left(1+\frac{1}{\sqrt{t}}\right)\left\|W_{0}\right\|_{L^{p}(\Sigma)} .
$$

2. There exists $C_{p}>0$ such that, for all $\psi_{0} \in L^{p}(\omega)$ :

$$
\forall t>0, \quad\left\|e^{t \Delta_{y}} \psi_{0}\right\|_{L^{\infty}(\omega)} \leq C_{p}\left(1+\frac{1}{\sqrt{t}}\right)\left\|\psi_{0}\right\|_{L^{p}(\omega)} .
$$

3. Let $W_{0} \in U C(\bar{\Sigma})$ be such that $\lim _{|x| \rightarrow+\infty} W_{0}(x, y)=0$. Then

$$
\lim _{t \rightarrow+\infty}\left\|e^{-t A} W_{0}\right\|_{L^{\infty}(\Sigma)}=0 .
$$

Vol. $14, n^{\circ} 4-1997$. 
Proof. - This lemma is well-known. Points 1 and 2 stem from the fact that (resp. $e^{t \Delta_{y}}$ ) is an analytic semigroup in every $L^{p}(\Sigma)$ [16]. As for Point 3, a proof involving detailed asymptotics of the fundamental solution can be found in [12]. Here is a quick - but less precise-argument.

Set $W(t)=e^{-t A} W_{0}$. The semigroup $e^{-t A}$ is a semigroup of contractions, therefore we assume that $W_{0}$ has compact support in $\bar{\Sigma}$; the result follows by density. Multiplication of the equation for $W$ by $W$ and integration yields

$$
\int_{\Sigma} W(t)^{2} d x d y+\int_{0}^{t} \int_{\Sigma}|\nabla W|^{2} d s d x d y=\int_{\Sigma} W_{0}^{2} d x d y
$$

From the maximum principle, identity (6.1) and parabolic estimates, the function $t \mapsto \int_{\Sigma}|\nabla W(t)|^{2} d x d y$ is uniformly continuous; therefore, from (6.1), it goes to 0 as $t \rightarrow+\infty$. Further, the function $t \mapsto \int_{\Sigma} W(t)^{2} d x d y$ goes to a limit as $t \rightarrow+\infty$, and there exists $\left(t_{n}\right)_{n}$ such that $\left(W\left(t_{n}\right)\right)_{n}$ converges in $H_{l o c}^{1}(\Sigma)$ to some function $W^{\infty}$. In view of the above remarks, $W^{\infty}$ is constant, and therefore $W^{\infty} \equiv 0$. This implies $\lim _{t \rightarrow+\infty}\|W(t)\|_{H^{1}(\Sigma)}=0$.

At this point, one only has to apply a classical bootstrap procedure to reach decay in $L^{\infty}$.

For the extinction results, we prove that $u(t):=\mathcal{S}(t) u_{0}$ falls under $\theta$ in finite time by following the idea of Aronson-Weinberger [2], [3]. As for the convergence part of Theorem 3.6, paragraph 1, we just have to examine the limiting equation for $u$ - as $x \rightarrow+\infty$ for large-but finite-time.

Proof of Theorem 3.4, part 1 . - The set of initial data $u_{0}$ will be chosen below a reference initial datum, denoted by $\bar{u}_{0}$, such that $(3.4)$ is true for $\bar{u}_{0}$, with given $L_{0}, \delta_{0}, \eta_{0}$ and $r_{0}$.

From the integral identity

$$
u(t)=u_{0}+\int_{0}^{t} e^{-(t-s) A} g(u(s)) d s,
$$

one may write, from Lemma 6.1 and for $p>N$ :

$$
u(t) \leq u_{0}+C\|g\|_{\text {Lip }} \int_{0}^{t}\left(1+\frac{1}{\sqrt{t-s}}\right)\left\|(u(s)-\theta)_{+}\right\|_{L^{p}(\Sigma)} d s .
$$

However we have, from (6.2) and the fact that is contracting in $L^{p}(\Sigma)$ :

$$
\left\|(u(t)-\theta)_{+}\right\|_{L^{p}(\Sigma)} \leq(L+2 \delta) \eta+\|g\|_{\text {Lip }} \int_{0}^{t}\left\|(u(s)-\theta)_{+}\right\|_{L^{p}(\Sigma)} d s,
$$


which implies, by Gronwall's Lemma: $\left\|(u(t)-\theta)_{+}\right\|_{L^{p}(\Sigma)} \leq(L+$ $2 \delta) \eta e^{\|g\|_{\mathrm{Lip}} t}$. To sum up, we have obtained the following inequality, for some large $C$ :

$$
u(t) \leq \bar{u}_{0}+C(L+2 \delta) \eta e^{\|g\|_{\mathrm{Lip}} t}
$$

From Lemma 6.1, there exists $t_{0}$ such that $e^{-t_{0} A} \bar{u}_{0} \leq \frac{\theta}{2}$; therefore, if we choose $(L+2 \delta) \eta \leq \frac{\theta}{2 C} e^{-\|g\|_{\text {Lip }} t_{0}}$ we have $u\left(t_{0}\right) \leq \theta$, and $u(t)=e^{-\left(t-t_{0}\right) A} u\left(t_{0}\right)$ for $t \geq t_{0}$. A second application of Lemma 6.1 yields Part 1 of Theorem 3.4.

Proof of Theorem 3.3, part 1. - Let $\bar{u}_{0} \in U C(\bar{\Sigma})$ be equal to 1 on $\Sigma_{L+\delta}$, and equal to $\theta-\eta$ on $\Sigma \backslash \Sigma_{L+2 \delta}$. Let $\bar{u}(t)$ be the solution of

$$
\bar{u}^{\prime}+A \bar{u}=\bar{g}(\bar{u}), \quad \bar{u}(0)=\bar{u}_{0},
$$

with $\bar{g}=0$ on $[0, \theta]$ and $\bar{g}=g$ on $[\theta, 1]$. Then $\mathcal{S}(t) u_{0} \leq \bar{u}$; moreover the same argument as above, applied to $\bar{u}(t)-\theta+\eta$, shows that $\bar{u}(t)$ falls under $\theta-\frac{\eta}{2}$ in tinite time, say, after $t_{0}$. Therefore, $\mathcal{S}(t) u_{0}$ is dominated, for $t \geq t_{0}$, by the solution of the equation $u^{\prime}=g(u), u\left(t_{0}\right)=\frac{\eta}{2}$, which converges exponentially to 0 .

To prove Part 1 of Theorem 3.6, we examine the asymptotic behaviour of the solutions of the problem

$$
\psi_{t}-\Delta_{y} \psi=g(\psi) \text { in } \omega \quad \partial_{\nu} \psi=0 \text { on } \partial \omega \text {. }
$$

Lemma 6.2. - Assume that $\psi(t, y)$ is a solution of $(6.4)$ with $\psi(0, y) \in$ $[0,1]$. Then $\psi(t, y)$ goes to a constant which either belongs to $[0, \theta]$ or which is equal to 1 .

Assume further that there exist $\omega^{\prime \prime} \subset \omega^{\prime} \subset \omega$ and $\left.\eta \in\right] 0, \theta[$ such that

$$
\psi(0) \geq \theta+\eta \text { in } \omega^{\prime \prime}, \quad \psi(0) \leq \theta-\eta \text { in } \omega \backslash \bar{\omega}^{\prime} .
$$

If $\left|\omega^{\prime \prime}\right|$ and $\left|\omega^{\prime} \backslash \omega^{\prime \prime}\right|$ are small enough, then $\psi(t)$ drops uniformly below $\theta$ in finite time. Conversely, if $\left|\omega \backslash \omega^{\prime \prime}\right|$ is small enough, then $\psi(t)$ goes to $I$ uniformly.

Proof. - The maximum principle and the Hopf lemma tell us that the only steady solutions of (6.4) are the constants in $[0, \theta]$ or 1 . Further, for every $\psi \in[0, \theta] \cup\{1\}$, we have $\lambda_{1}\left(-\Delta_{y}-g^{\prime}(\psi)\right) \geq 0$. Since $0 \leq \psi(0) \leq 1$, the Vol. $14, n^{\circ}$ 4-1997. 
$\omega$-limit-set of $\psi(0)$ is nonempty; further, Theorem $I I .1$, part (ii) of Lions [21] asserts that it is a singleton.

Therefore, it only remains to give sufficient conditions ensuring that $\psi(t)$ will either converge to 1 , or to some constant less than $\theta$, but this is not too difficult: indeed, if $\left|\omega^{\prime \prime}\right|$ and $\left|\omega^{\prime} \backslash \omega^{\prime \prime}\right|$ are small enough, the same argument as in the proof of Theorem 3.4, part 1 proves that $\psi(t)$ drops uniformly below $\theta$ in finite time. On the other hand, if $\left|\omega \backslash \omega^{\prime \prime}\right|$ is small enough, then $<\psi(0)>\geq \theta+\frac{\eta}{2}$, and since $\psi(t) \geq e^{t \Delta_{y}} \psi(0)$, then $\psi(t) \geq \frac{\eta+3 \theta}{4}$ for large enough $\ell$.

Proof of Theorem 3.6, part 1. - Straightforward in view of Lemma 6.2: if $\left|\omega^{\prime \prime}\right|$ and $\left|\omega^{\prime} \backslash \omega^{\prime \prime}\right|$ are small enough, it is possible to construct $\bar{\psi}_{0} \geq u_{0}$ satisfying (6.5) - possibly with different $\omega^{\prime}$ and $\omega^{\prime \prime}$ - such that the solution $\bar{\psi}(t)$ of (6.4) with initial datum $\bar{\psi}_{0}$ will eventually drop below $\theta_{0}$ and extinction will occur. Conversely, if $|\omega| \omega^{\prime \prime} \mid$ is small enough, then by Lemma 6.2 once again: $\lim _{t \rightarrow+\infty}\left(\liminf _{x \rightarrow+\infty} u(t, x, y)\right)=1$; therefore $\mathcal{S}\left(t_{0}\right) u_{0}$ satisfies the assumptions of Theorem 3.2 for $t_{0}>0$ large enough.

\section{AN ELLIPTIC PROBLEM IN A FINITE CYLINDER}

If we wish to prove propagation theorems in Case $\mathrm{A} 2$, we have, as it will be clear later, to prove that the solution goes to 1 somewhere. This can be done very quickly by noticing that the solution of the ODE $\dot{u}=g(u), u(0)>\theta$ goes to 1 as $t \rightarrow+\infty$. It is also possible to take a much longer way, which gives us an insight in the solutions of the steady problem in a finite strip. This insight is of independent interest, and this is why we are going to spend some time on it now. For $L>0$ let us consider the problem

$$
\begin{aligned}
-\Delta \psi+\alpha(y) \psi_{x} & =g(\psi) \quad \text { in } \Sigma_{L} \\
\frac{\partial \psi}{\partial \nu} & =0 \quad \text { on }]-L, L[\times \partial \omega \\
\psi( \pm L, y) & =0 \quad \text { in } \omega
\end{aligned}
$$

Let us notice that, trivially, all the solutions of (7.1) remain between 0 and 1; this will not be said again. Let us also point out that, in one space dimension, the problem is fully understood; see [30], Chapter 13.

For a solution $\psi$ of $(7.1)$, we set

$$
\mu_{1}(\psi):=\mu_{1}\left(-\Delta+\alpha(y) \partial_{x}-g^{\prime}(\psi)\right)
$$


THEOREM 7.1. - Assume $g$ satisfies the assumptions of case A2, and that $\alpha$ has zero mean. For $L>0$ large enough, Problem (7.1) has at least three nonnegative solutions: the zero solutions, and two positive solutions $\psi_{L}^{-}<\psi_{L}^{+}$, which additionally satisfy $\mu_{1}(0)>0, \mu_{1}\left(\psi_{L}^{-}\right)<0$ and $\mu_{1}\left(\psi_{L}^{+}\right)>0$. Finally there holds, uniformly on compact subsets of $\bar{\Sigma}$ :

$$
\lim _{L \rightarrow+\infty} \psi_{L}^{-}=\theta, \quad \lim _{L \rightarrow+\infty} \psi_{L}^{+}=1
$$

An open problem is the number of solutions between 0 and $\psi_{L}^{+}$.

Only the "ignition temperature" case is be treated, once again because this is really the one we have in mind. The bistable case will be examined somewhere else.

Adapting the method of [6], we see that there exists a unique solution $\psi^{\infty}$ to the problem

$$
\begin{aligned}
-\Delta \psi+\alpha(y) \psi_{x} & =g(\psi) & & \text { in } \Sigma_{+} \\
\frac{\partial \psi}{\partial \nu} & =0 & & \text { on } \mathbb{R}_{+} \times \partial \omega \\
\psi(0, y) & =0 & & \psi(+\infty, y)=1
\end{aligned}
$$

Moreover, $\psi_{x}^{\infty}>0$ in $\bar{\Sigma}$. In the same vein, there exists a unique $\tilde{\psi}^{\infty}$ solution of the same PDE as in (7.2), but posed in $\Sigma_{-}$, and such that $\tilde{\psi}^{\infty}(-\infty, y)=1, \psi^{\infty}(0, y)=0$. In this case we have $\tilde{\psi^{\infty}}{ }_{x}<0$. This implies the two following lemmas, that will be useful to us in the proof of Theorem 7.1.

LEMMA 7.2. - Let $\left(\psi_{L}\right)_{L}$ be a branch of nontrivial solutions of (7.1) in $\Sigma_{L}$. Then one of the two statements holds.

1. There exists $\left(L_{n}\right)_{n}$ going to $+\infty$, such that, uniformly on $\bar{\Sigma}_{L_{n}}$, there holds

$$
\limsup _{n \rightarrow+\infty} \psi_{L_{n}} \leq \theta
$$

In this case, there exists an interval $I_{n}$ of $]-L_{n}, L_{n}\left[\right.$, with $\lim _{L \rightarrow+\infty}\left|I_{n}\right|=+\infty$, such that $\psi_{L_{n}}$ tends to $\theta$ on $I_{n} \times \bar{\omega}$. Furthermore, $\mu_{1}\left(\psi_{L_{n}}\right)<0$.

2. There holds $\tau_{-L} \psi_{L} \rightarrow \psi_{\infty}$, uniformly on the compact subsets of $\bar{\Sigma}_{+}$. In this case, there exists $x(L)>0$, such that $\min (|x(L)-L|,|x(L)+L|)$ is a bounded function of $L$, and such that there holds

$$
\bar{\Sigma}_{x(L)} \subset\left\{(x, y) \in \bar{\Sigma}: \psi_{L} \geq \frac{1+\theta}{2}\right\} .
$$

Finally, there exists $\delta>0$ such that $\mu_{1}\left(\psi_{L}\right) \geq \delta$. 
Proof. - Let the assumption of Statement 1 hold. In this case, there exists $\left(x_{n}, y_{n}\right) \in \bar{\Sigma}_{L_{n}}$ such that $\psi_{L_{n}}\left(x_{n}, y_{n}\right)>\theta$. By elliptic estimates, and because $g(\theta)=0$, there exists two sequences $\varepsilon_{n} \rightarrow 0$ and $a_{n} \rightarrow+\infty$ such that $\psi_{L_{n}} \geq \theta-\varepsilon_{n}$ on $\left[x_{n}-a_{n}, x_{n}+a_{n}\right] \cap \bar{\Sigma}_{L_{n}}$, which proves the existence of the interval $I_{n}$. To end the proof of statement 1., we use a very classical argument: let us indeed recall that the assumptions on $g$ imply the existence of $\eta_{0}>0$ such that $g(u) \leq u g^{\prime}(u)$ on the interval $\left[0, \theta+\eta_{0}\right]$. Therefore the function $u:=\psi_{L_{n}}>0$ satisfies

$$
\begin{array}{rlrl}
-\Delta u+\alpha(y) u_{x} & \leq g^{\prime}\left(\psi_{L_{n}}\right) u \quad & \text { in } \Sigma_{L} \\
\frac{\partial u}{\partial \nu} & =0 & & \text { on }]-L, L[\times \partial \omega \\
u( \pm L, y) & =0 & & \text { in } \omega
\end{array}
$$

which implies $\mu_{1}\left(\psi_{L_{n}}\right)<0$.

If now the assumption of Statement 1 does not hold, there exists $\theta_{1}>0$ independent of $L$, and $\left(x_{L}, y_{L}\right) \in \bar{\Sigma}_{L}$ such that $\psi_{L}\left(x_{L}, y_{L}\right) \geq \theta_{1}$. From now on, we denote by $x_{L}$ the smallest $x$ for which there exists $y$ such that $\psi_{L}(x, y)=\theta_{1}$. We know that the quantity $\left|x_{L}+L\right|$ is bounded; otherwise there would exist a sequence $L_{n} \rightarrow+\infty$ such that $\left(\tau_{x_{L_{n}}} \psi_{L_{n}}\right)_{n}$ would converge to $\psi_{\infty}$ solution of (7.1), but this time in $\Sigma$, with $\psi_{\infty}\left(0, y_{\infty}\right)=\theta_{1}$, for some $y_{\infty} \in \bar{\omega}$. By elliptic regularity, $\left\|\psi_{\infty}\right\|_{C^{2}(\bar{\Sigma})}<+\infty$. Let $a<0<b$; integrating the equation for $\psi_{\infty}$ on $[a, b] \times \omega$ we get

$$
\begin{aligned}
\int_{a}^{b} \int_{\omega} g\left(\psi_{\infty}\right) d x d y= & \int_{\omega} \alpha(y)\left(\psi_{\infty}(b, y)-\psi_{\infty}(a, y)\right) d y \\
& +\int_{\omega}\left(\partial_{x} \psi_{\infty}(b, y)-\partial_{x} \psi_{\infty}(a, y)\right) d y
\end{aligned}
$$

which proves that $g\left(\psi_{\infty}\right) \in L^{1}(\Sigma)$. Therefore, multiplying the equation for $\psi_{\infty}$ and integrating we see that $\nabla \psi_{\infty} \in L^{2}(\Sigma)$, which in turn implies, by regularity, that $\left|\nabla \psi_{\infty}\right|$ goes to 0 as $|x| \rightarrow+\infty$, uniformly in $y \in \bar{\omega}$. By compactness there exist two sequences $a_{n} \rightarrow-\infty$ and $b_{n} \rightarrow+\infty$ such that

$$
\lim _{n \rightarrow+\infty} \psi_{\infty}\left(a_{n}, y\right)=\psi_{-}(y), \quad \lim _{n \rightarrow+\infty} \psi_{\infty}\left(b_{n}, y\right)=\psi_{+}(y)
$$

in $C^{1}(\bar{\omega})$; since $\nabla \psi_{\infty}$ vanishes as $|x| \rightarrow+\infty$ the functions $\psi_{ \pm}(y)$ are constants, which we still denote by $\psi_{ \pm}$. 
Now, the integral identity for $g(\psi)$, with $a=a_{n}$ and $b=b_{n}$ yields, for large $n$ :

$$
\int_{a_{n}}^{b_{n}} \int_{\omega} g\left(\psi_{\infty}\right) d x d y=\int_{\omega} \alpha(y)\left(\psi_{+}-\psi_{-}\right) d y+o(1)
$$

letting $n \rightarrow+\infty$ and taking into account $\langle\alpha>=0$ we get: $\int_{\Sigma} g(\psi) d x d y=0$. As a consequence, either $\psi \leq \theta$ or $\psi \equiv 1$; the two possibilities being absurd due to the fact that $\psi_{\infty}\left(0, y_{\infty}\right)=\theta_{1}$.

The uniqueness of the solution $\psi^{\infty}$ to (7.2) implies exactly that $\tau_{L} \psi_{L} \rightarrow \psi$, uniformly on the compact subsets of $\bar{\Sigma}_{+}$.

Let us now prove the assertion on the set $\left\{\psi_{L}>\frac{1+\theta}{2}\right\}$. If this were not true, there would exist a sequence $L_{n} \rightarrow+\infty$, and a sequence $\left(x_{n}, y_{n}\right)_{n}$ of $\bar{\Sigma}_{L_{n}}$ such that $\min \left(\left|x_{n}-L_{n}\right|,\left|x_{n}+L_{n}\right|\right)$ tends to $+\infty$, and such that

$$
\psi_{L_{n}}\left(x_{n}, y_{n}\right)=\frac{1+\theta}{2} \text {. }
$$

Letting $n \rightarrow+\infty$ and arguing as above on the sequence $\tau_{x_{n}} \psi_{L_{n}}$, we end up with a nonconstant solution of (7.1), but this time on the whole cylinder $\Sigma$. This is absurd because $\alpha$ has zero mean.

It remains to prove that $\mu_{1}\left(\psi_{L}\right)$ is controlled from below. We recall that there exists $M>0$ such that $-M \leq \mu_{1}\left(\psi_{L}\right) \leq M$. Let us introduce an associated eigenfunction $u_{L}$, and let $\left(\tilde{x}_{L}, \tilde{y}_{L}\right)$ be defined such that

$$
u_{L}\left(\tilde{x}_{L}, \tilde{y}_{L}\right)=\left\|u_{L}\right\|_{L^{\infty}(\Sigma)}=1
$$

There exists a sequence $L_{n} \rightarrow+\infty$ such that $\left(\tau_{x_{L_{n}}} u\right)_{n}$ converges in $C_{\text {loc }}^{2}$, as $n \rightarrow+\infty$, towards a function $u_{\infty}$; moreover $\mu_{1}\left(\psi_{L_{n}}\right) \rightarrow \mu_{\infty}$. Two cases have to be examined.

Case 1. For a subsequence still denoted by $\left(L_{n}\right)_{n}$, the quantity $\min \left(x_{L_{n}}+L_{n}, L_{n}-x_{L_{n}}\right)$ tends to $+\infty$. In this case we have

$$
\begin{aligned}
-\Delta u_{\infty}+\alpha(y) \partial_{x} u_{\infty}-g^{\prime}(1) u_{\infty} & =\mu_{\infty} u_{\infty} \quad \text { in } \Sigma \\
\frac{\partial u_{\infty}}{\partial \nu} & =0 \quad \text { on } \mathbb{R} \times \partial \omega
\end{aligned}
$$

which obviously implies $u_{\infty} \equiv 1$ and $\mu_{\infty}=-g^{\prime}(1)$.

Vol. 14, n ${ }^{\circ}$ 4-1997. 
Case 2. the quantity $\min \left(x_{L}+L, L-x_{L}\right)$ is bounded. Assume that the first argument of the min is bounded; in this case we have

$$
\begin{aligned}
-\Delta u_{\infty}+\alpha(y) \partial_{x} u_{\infty}-g^{\prime}\left(\psi^{\infty}\right) u_{\infty} & =\mu_{\infty} u_{\infty} \quad \text { in } \Sigma_{+} \\
\frac{\partial u_{\infty}}{\partial \nu} & =0 \quad \text { on } \mathbb{R}_{+} \times \partial \omega
\end{aligned}
$$

Because $\psi_{x}>0$ in $\bar{\Sigma}$, an argument similar to the one in [7], Proposition 5.7 , shows that $\mu_{\infty}>0$. Finally, in case the second argument of the min is bounded, one only has to introduce the solution $\tilde{\psi}$ of $(7.2)$, but this time in $\Sigma_{-}$.

LEMMA. - Let $\theta_{1}>\theta$ be fixed. For $L_{0}>0$ large enough, there exists at most one solution $\psi_{L}$ of (7.1) such that

$$
\max _{y \in \bar{\omega}} \psi_{L}(0, y) \geq \theta_{1}
$$

If such a branch $\left(\psi_{L}\right)_{L}$ exists, it satisfies Statement 1 of Lemma 7.2.

Proof. - Assume that there is a sequence $L_{n} \rightarrow+\infty$ such that there exist $\psi_{L_{n}} \neq \tilde{\psi}_{L_{n}}$ satisfying the assumptions of the lemma. Set

$$
\zeta_{n}:=\frac{\psi_{L_{n}}-\tilde{\psi}_{L_{n}}}{\left\|\psi_{L_{n}}-\tilde{\psi}_{L_{n}}\right\|_{L^{\infty}\left(\Sigma_{L_{n}}\right)}}
$$

Let $\left(x_{n}, y_{n}\right) \in \bar{\Sigma}$ be such that $\zeta_{n}\left(x_{n}, y_{n}\right)=1$. The proof of the lemma can be obtained by deriving an equation for $\tau_{\boldsymbol{x}_{n}} \zeta_{n}$, and arguing as in the end of the proof of Lemma 7.2.

Proof of Theorem 7.1. - It is based on the construction of a sub-solution.

1. For $\left.\theta_{1} \in\right] \theta, 1\left[\right.$ and $b>0$, there exists a solution $\underline{\psi}_{2}(x, y)$ of

$$
\begin{aligned}
-\Delta \psi+\alpha(y) \psi_{x} & =g(\psi) \quad \text { in } \Sigma_{b} \\
\frac{\partial \psi}{\partial \nu} & =0 \quad \text { on }]-b, b[\times \partial \omega \\
\psi( \pm b, y) & =\theta_{1} \quad \text { in } \omega
\end{aligned}
$$

Indeed $\theta_{1}$ is a subsolution to (7.4), and 1 is a supersolution.

2. For $\delta>0$, we now want to solve the following problem:

$$
\begin{aligned}
-\Delta \psi+\alpha(y) \psi_{x} & =0 & & \text { in } \Sigma_{-} \\
\frac{\partial \psi}{\partial \nu} & =0 & & \text { on } \mathbb{R}_{-} \times \partial \omega \\
\psi(0, y) & =\theta_{1}, & & \lim _{x \rightarrow-\infty} \frac{\psi(x, y)}{x}=\delta
\end{aligned}
$$


Let us first seek solutions of (7.5) - except the condition at $x=0$ - of the form $e_{\delta}(x, y)=\delta x+\phi_{\delta}(y)$. Obviously, for every given $\delta>0$, there exists a unique solution of that form, since $\phi_{\delta}$ has to solve $-\Delta_{y} \phi_{\delta}+\delta \alpha(y)=0$ in $\omega$, with Neumann boundary conditions (Recall that $\langle\alpha\rangle=0$ ). Now, for $A>0$, set $\bar{\psi}(x, y)=e_{\delta}(x, y)+A, \underline{\psi}(x, y)=e_{\delta}(x, y)-A$. For $A$ large enough, $\bar{\psi}$ (resp. $\psi$ ) is a supersolution (resp. a subsolution) of (7.5). A straightforward adaptation of the method of sub and supersolutions solve (7.5) on $]-a, 0\left[\times \omega\right.$ first, with $\psi(-a, y)=e_{\delta}(-a, y)$; then pass to the limit $a \rightarrow+\infty$ - yields the existence of a solution of (7.5), which will be denoted by $\psi^{\delta}$.

Let us now prove that

$$
\lim _{\delta \rightarrow 0}\left\|\partial_{x} \psi^{\delta}(0, .)\right\|_{L^{\infty}(\omega)}=0
$$

By Theorem 5.6 of [1], Theorem 2.3 of [9] and the fact that $e_{\delta}-A \leq \psi^{\delta} \leq$ $e_{\delta}+A$ for large $A$, there exists $\varepsilon>0$ such that the following asymptotics hold, uniformly in $y \in \bar{\omega}$ :

$$
\begin{aligned}
\psi^{\delta}(x, y) & =\delta x+\phi_{\delta}(y)+O\left(e^{\varepsilon x}\right) \\
\partial_{x} \psi^{\delta}(x, y) & =\delta+O\left(e^{\varepsilon x}\right)
\end{aligned} \quad \text { as } x \rightarrow-\infty .
$$

This proves, in particular, that $\partial_{x} \psi^{\delta}>0$. Now, let $\left(\delta_{n}\right)_{n}$ be a sequence going to 0 . There exists a subsequence - still denoted by $\left(\delta_{n}\right)_{n}$ - such that $\psi^{\delta_{n}} \rightarrow \psi^{\infty}$ in $C^{1}(K)$, for every compact $K \subset \bar{\Sigma}_{-}$. Notice that the corner $\{0\} \times \partial \omega$ is not too annoying because of the Neumann boundary condition, which allows a local extension by reflection of $\psi^{\delta}$, and so, in particular, convergence in $\{0\} \times \bar{\omega}$. The function $\psi^{\infty}$ is still $x$-nondecreasing, therefore it has a limit $l(y)$ as $x \rightarrow-\infty$. However, in view of Theorem 5.6 of [1], this limit has to satisfy $-\Delta_{y} l=0$ in $\omega$ with Neumann boundary conditions; hence $l(y) \equiv$ Constant $:=l$. Let us prove that $l=\theta_{1}$. If it were not true, then $\partial_{x} \psi^{\infty}>0$. In view of Theorem 5.6 of [1] and Theorems 2.1 and 2.3 of [9], $\psi^{\infty}$ would have the following asymptotics:

$$
\psi^{\infty}(x, y)=l+e^{\lambda_{-} x}\left(x^{p} \phi_{-}(y)+o\left(x^{p}\right)\right) \quad \text { as } x \rightarrow-\infty .
$$

The function $e^{\lambda_{-} x} \phi_{-}(y)$ is an exponential solution of the first two equations of (7.5), and therefore changes sign if nonzero. Since $\partial_{x} \psi^{\infty}>0$, this would mean that in fact $\phi_{-} \equiv 0$, and therefore that $\psi^{\infty}-l$ would decay faster than any exponential. However, for $\varepsilon \in] 0, \theta_{1}[$ and $M>0$ large enough, the function $\underline{e}(x, y):=\varepsilon e^{M x}$ satisfies

$$
\begin{aligned}
-\Delta \underline{e}+\alpha(y) \underline{e}_{x} & =\left(-M^{2}+M \alpha(y)\right) \underline{e} \leq 0 \quad \text { in } \Sigma_{-} \\
\frac{\partial \underline{e}}{\partial \nu} & =0 \quad \text { on } \mathbb{R}_{-} \times \partial \omega \\
\underline{e}(0, y) & <\theta_{1}, \quad \lim _{x \rightarrow-\infty} e(x, y)=0
\end{aligned}
$$

Vol. 14, n 4-1997. 
Therefore we would have $l+\underline{e} \leq \psi^{\infty}$, a contradiction. As a consequence $l=\theta_{1}$, and $\psi^{\infty} \equiv \theta_{1}$. This implies (7.6).

3. We are now in a position to construct a weak subsolution. The function $\underline{\psi}_{2}$ of paragraph 1 of this proof is such that there exists $\mu>0$ such that $\partial_{x} \underline{\psi}_{2}(-b, y) \geq 2 \mu, \partial_{x} \underline{\psi}_{2}(b, y) \leq-2 \mu$. From our considerations of paragraph 2 of the present proof, there exist $\underline{\psi}_{1}(x, y)$ (resp. $\underline{\psi}_{3}(x, y)$ ) solution of

$$
\begin{aligned}
-\Delta \psi+\alpha(y) \psi_{x} & =0 \quad \text { in }]-\infty,-b[\times \omega(\text { resp. }] b,+\infty[\times \omega) \\
\frac{\partial \psi}{\partial \nu} & =0 \quad \text { on }]-\infty,-b[\times \partial \omega \text { (resp. }] b,+\infty[\times \partial \omega) \\
\psi(-b, y) & \left.=\theta_{1} \text { (resp. } \psi(b, y)=\theta_{1}\right) \\
\lim _{x \rightarrow-\infty} \frac{\psi(x, y)}{x} & >0\left(\text { resp. } \lim _{x \rightarrow+\infty} \frac{\psi(x, y)}{x}<0\right)
\end{aligned}
$$

which additionally satisfy: $\partial_{x} \psi_{1}(-b, y) \leq \mu, \partial_{x} \psi_{3}(b, y) \geq-\mu$. Further, the set $\left\{\psi_{1}(x, y)=0\right\}$ (resp. $\left\{\psi_{3}(x, y)=0\right\}$ ) has the form $\left\{\left(x_{1}(y), y\right)\right.$, $y \in \bar{\omega}\}$ (resp. $\left.\left.\left\{x_{3}(y), y\right), y \in \bar{\omega}\right\}\right)$, where $x_{i}(y)$ belong to $C^{1}(\bar{\omega})$ and have $x_{1}(y)<-b, x_{3}(y)>b$, for all $y \in \bar{\omega}$.

Now, the function $\underline{\psi}_{L}(x, y)$ defined by

$$
\begin{aligned}
0 & \text { in }\left\{(x, y): x \leq x_{1}(y), y \in \omega\right\} \\
\psi_{1}(x, y) & \text { in }\left\{(x, y): x_{1}(y) \leq x \leq-b, y \in \omega\right\} \\
\psi_{2}(x, y) & \text { in } \Sigma_{b} \\
\underline{\psi}_{3}(x, y) & \text { in }\left\{(x, y): b(y) \leq x \leq x_{3}(y), y \in \omega\right\} \\
0 & \text { in }\left\{(x, y): x_{3}(y) \leq x, y \in \omega\right\}
\end{aligned}
$$

is a weak subsolution to the stationary problem corresponding to (1.1), which is in addition $\leq \theta_{2}$.

4. Let us notice that, at this stage, we have not proved yet any information that is valuable enough to justify the above long developments. Let us do it now by examining the implications (7.7). Fix $L_{0}>0$ such that $\underline{\psi}_{L_{0}}$ can be defined by (7.7). For every $L>L_{0}$, the function $\psi_{L_{0}}$ - suitably extended by 0 outside $\Sigma_{L_{0}}$ - is a subsolution to (7.1); therefore for every $L>L_{0}$ there exists $\psi_{L}^{+}>\underline{\psi}_{L_{0}}$ solution of (7.1), such that $\mu_{1}\left(\psi_{L}^{+}\right) \geq 0$. Because of Lemma $7.1, \mu_{1}\left(\psi_{L}^{+}\right)>\delta$ for $L>L_{0}$ large enough, and the asymptotic behaviour of $\psi_{L}^{+}$is obvious.

Finally, let us deal with $\psi_{L}^{-}$, by a claśsical degree argument - cf [30], Chap. 13 -. We transform $\Sigma_{L}$ into a fixed domain, and make $L$ a bifurcation parameter via the change of variables $x \mapsto \frac{x}{L}$. Set

$$
E=\left\{u \in C\left(\bar{\Sigma}_{1}\right), u(-1, .)=u(1, .)=0 ;-1<u<1\right\}
$$


Problem (7.1) may be written as $F(L, u)=0, F$ being a locally compact map from $\mathbb{R}_{+} \times E$ to $E$. Let $L>0$ be chosen so that $\psi_{L}^{+}$exists. Let $\Omega_{0}$ be the ball of $E$ with centre 0 and radius $\varepsilon$, and let $\Omega_{+}$be the ball of $E$ with centre $\psi_{+}^{L}$ and radius $\varepsilon$. For $\varepsilon>0$ small enough, 0 (resp. $\psi_{L}^{+}$) is the only solution of $F(L, u)=0$ in $\bar{\Omega}_{0}$ (resp. $\bar{\Omega}_{+}$), and we have

$$
\operatorname{deg}\left(F(L, .), \Omega_{0}, 0\right)=\operatorname{deg}\left(F(L, .), \Omega_{+}, 0\right)=1 .
$$

For $L>0$ small enough, we have $\operatorname{deg}(F(L,), E, 0)=$.1 ; therefore, by invariance of the degree under homotopy, there exists a solution $\psi_{L}^{-} \in E \backslash\left(\Omega_{0} \cup \Omega_{+}\right)$such that $\mu_{1}\left(\psi_{L}^{-}\right) \leq 0$. The asymptotic behaviour of $\psi_{L}^{-}$is given by the combination of Lemmas 7.2 and 7.3 .

\section{CREATION OF TWO FRONTS}

A key ingredient is the time-exponential convergence to 1 on every subset of the form $\left\{(t, x): \tilde{c}^{\prime} t \leq x \leq c^{\prime} t\right\} \times \omega$, with $\tilde{c}<\tilde{c}^{\prime}<c^{\prime}<c$. In [11], Fife and McLeod proceed by sub and supersolutions, and we imitate them. To make the notations simpler we assume, until the end of the paragraph, and without loss of generality, that $\langle\alpha\rangle=0$. This implies $\tilde{c}<0<c$.

The sub and supersolutions can readily be constructed as in [11] for Case A1; and a little less easily for Case A2. A perturbation argument is then used.

\subsection{Sub and supersolutions}

Let us first deal with case A1. As said above, Lemma 8.1 below can be proved as in [11], Lemma 6.1, and its proof will not be given.

LemMa 8.1. - Let $u_{0}$ be as in Theorem 3.3. Then, when $L$ is large enough, there exist constants $x_{1}, x_{2}, q>0, \omega>0$ such that

$\tau_{x_{1}+c t} \phi+\tau_{x_{1}+\tilde{c} t} \tilde{\phi}-1-q e^{-\omega t} \leq \mathcal{S}(t) u_{0} \leq \tau_{x_{2}+c t} \phi+\tau_{x_{2}+\tilde{c} t} \tilde{\phi}-1+q e^{-\omega t}$

As for case A2, we first need the analogue of Lemma 4.2; this is unfortunately a little more intricate. We set $\tilde{w}_{r}(x)=w_{r}(-x)$, for every $r>0$.

LEMma 8.2. - Let $u_{0}$ be as in Theorem 3.4 and L be large enough so that Theorem 7.1 applies. There exist $x_{1}, x_{2}, q>0, \omega>0$ and $t_{0}>0$ such Vol. 14. $n^{\circ} 4-1997$. 
that, for all $t \geq t_{0}$, there holds:

(8.1) $\tau_{x_{1}+c t} \phi+\tau_{x_{1}+\tilde{c} t} \tilde{\phi}-\inf \left(\frac{q}{\tau_{x_{1}+c t} w_{\frac{r}{2}}} e^{-\omega t}, \frac{q}{\tau_{x_{1}+\tilde{c} t} \tilde{w}_{\frac{r}{2}}} e^{-\omega t}\right)$

$\leq \mathcal{S}(t) u_{0}+1 \leq \tau_{x_{2}+c t} \phi+\tau_{x_{2}+\tilde{c} t} \tilde{\phi}+\inf \left(\frac{q}{\tau_{x_{2}+c t} w_{\frac{r}{2}}} e^{-\omega t}, \frac{q}{\tau_{x_{2}+\tilde{r} t} \tilde{w}_{\frac{r}{2}}} e^{-\omega t}\right)$

Proof.

1. We first prove the upper bound. The same method as in Lemma 4.1 yields the existence of $x_{2}$ such that

$$
\mathcal{S}(t) u_{0} \leq \phi\left(x+x_{2}+c t, y\right)+q / w_{\frac{r}{2}}(x) e^{-\omega t} .
$$

By possibly taking $x_{2}$ large enough, we can also show:

$$
\mathcal{S}(t) u_{0} \leq \tilde{\phi}\left(x+x_{2}+\tilde{c} t, y\right)+q / w_{\frac{r}{2}}(-x) e^{-\omega t} .
$$

The infimum of the right handside of the above two estimates is dominated by the right handside of (8.1), by estimates (2.2.b).

2. The next four steps are devoted to the left handside. Let us first prove that $\mathcal{S}(t) u_{0}$ tends to 1 uniformly on the compact subsets of $\bar{\Sigma}$, as long as $L$ is large enough. Choose $\left.\theta_{2} \in\right] \theta, \theta+\eta\left[\right.$ and a subsolution $\underline{\psi} \leq \theta_{2}$ as in (7.7). When $L>0$ is large enough, $\underline{\psi} \leq u_{0}$; therefore $\mathcal{S}(t) \underline{\psi} \leq \mathcal{S}(t) u_{0}$. The function $\mathcal{S}(t) \psi$ is time-increasing - because $\psi$ is a subsolution -; as a consequence, by standard $C^{2}$ estimates, it converges to a solution $\psi$ of

$$
\begin{aligned}
-\Delta \psi+\alpha(y) \partial_{x} \psi & =g(\psi) \quad \text { in } \Sigma \\
\frac{\partial \psi}{\partial \nu} & =0 \quad \text { on } \mathbb{R} \times \partial \omega
\end{aligned}
$$

on every compact of $\bar{\Sigma}$. By the same arguments as in the proof of Lemma 7.2, we can only have $\psi \equiv 1$.

3. Define the functions $w(x, y)$ and $\tilde{w}(x, y)$ as in (5.8), by

$$
w(x, y)=e^{\rho x} \psi_{-}(y), \quad \tilde{w}(x, y)=e^{-\tilde{\rho} x} \tilde{\psi}_{-}(y)
$$

the functions $\psi_{-}$and $\tilde{\psi}_{-}$being positive solutions of

$$
(8.2)-\Delta_{y} \psi+r\left(\alpha(y)-\langle\alpha>+\varepsilon \eta) \psi=r^{2} \psi \text { in } \omega, \quad \frac{\partial \psi}{\partial \nu}=0 \text { on } \partial \omega\right.
$$

with $\eta$ small enough and $\varepsilon=1, r=\rho$ (resp. $\varepsilon=-1, r=-\ddot{\rho}$ ) if $\psi=\psi_{-}$(resp. $\psi=\tilde{\psi}_{-}$). They will be chosen so that $\left\|\psi_{-}\right\|_{\infty}<1$. (resp. 
$\left.\left\|\tilde{\psi}_{-}\right\|_{\infty}<1.\right)$. Select $\left.\theta_{1} \in\right] 0, \frac{\theta}{4}\left[\right.$ such that $-g^{\prime} \geq-\frac{g^{\prime}(1)}{2}$ in $\left[1-4 \theta_{1}, 1\right]$ and choose $A>0$ once and for all such that

$$
\begin{aligned}
& \forall \zeta \leq-A+1, \quad \phi(\zeta, y) \leq \theta_{1}, 1-\tilde{\phi}(\zeta, y) \leq \theta_{1} \\
& \forall \zeta \geq A-1, \quad \tilde{\phi}(\zeta, y) \leq \theta_{1}, 1-\phi(\zeta, y) \leq \theta_{1}
\end{aligned}
$$

In the whole sequel, we will set $\zeta=x+M+c t$, and $\tilde{\zeta}=x-M+\tilde{c} t$, with $M>0$ to be chosen later. We require the functions $w(\zeta-A, y)$ and $\tilde{w}(\tilde{\zeta}-A, y)$ to be such that

$$
(w(\zeta-A, y)=\tilde{w}(\tilde{\zeta}-A, y)) \quad \Longrightarrow \quad(w(\zeta-A, y)>1)
$$

It is enough to ask (8.3.b) to hold for $t=0$; this is equivalent to impose:

$$
\inf _{y \in \omega}\left(\psi_{-}(y)\left(\frac{\tilde{\psi}_{-}(y)}{\psi_{-}(y)}\right)^{\frac{1}{\rho+\tilde{\rho}}}\right) e^{\frac{2 \tilde{\rho}}{\rho+\tilde{\rho}} M}>1 .
$$

Under this condition, there exist two functions $X_{1}(t, y)<X_{2}(t, y)$ such that the following holds:

(8.3.d)

$$
\inf (1, w(\zeta-A), \tilde{w}(\tilde{\zeta}-A))=\begin{gathered}
w(\zeta-A) \text { if } x \leq X_{1}(t, y) \\
1 \text { if } x \in\left[X_{1}(t, y), X_{2}(t, y)\right] \\
\tilde{w}(\tilde{\zeta}-A) \text { if } x \geq X_{2}(t, y)
\end{gathered}
$$

We set $\varphi(t, x, y)=\inf (1, w(\zeta-A), \tilde{w}(\tilde{\zeta}-A))$. Finally, we require in addition that

$$
M>2 A+2
$$

4. We look for a subsolution of (1.1) in the form

$$
\underline{u}(t, x)=\phi(\zeta+\xi(t), y)+\tilde{\phi}(\tilde{\zeta}+\tilde{\xi}(t), y)-1-q(t) \varphi(t, x, y) .
$$

We furthermore require the following conditions to hold:

$$
\begin{array}{ll}
\text { (C1) } & q(t)>0, \quad q^{\prime}(t)<0, \\
\text { (C2) } & \xi^{\prime}(t)<0, \quad \tilde{\xi}^{\prime}(t)>0, \\
\text { (C3) } & \xi(0)=\tilde{\xi}(0)=0, \quad|\xi(t)| \leq 1, \quad|\tilde{\xi}(t)| \leq 1 .
\end{array}
$$

Denote by $\mathcal{N}$ the nonlinear operator $\mathcal{N} u=u_{t}-\Delta u+\alpha(y) u_{x}-g(u)$. The way to construct $q, \xi$ and $\tilde{\xi}$ is now standard: assume that conditions (C1)-(C3) hold, and estimate $\mathcal{N} \bar{u}$ with barehands. 
Two parameters will need special attention: the initial value of $q$, denoted by $\varepsilon$, and the real number $M$. Therefore, in the whole sequel, $C$ will denote a nonnegative constant which does no depend on these parameters. Three cases are to be examined.

Case 1. $\zeta \leq-A$.

This means that $\dot{\zeta} \leq-A$ as well. In view of the definition of $\theta_{1},(8.2)$, (8.3) and (C3), we have $\varphi(t, x, y)=w(\zeta-A, y)$ and

$$
\begin{aligned}
\mathcal{N} \underline{u}= & \xi^{\prime}(t) \phi_{x}(\zeta+\xi(t), y)+\tilde{\xi}^{\prime}(t) \tilde{\phi}_{x}(\tilde{\zeta}+\tilde{\xi}(t), y) \\
& -\left(q^{\prime}(t)+\rho(c-\eta) q\right) w(\zeta-A, y)+g(\tilde{\phi}(\tilde{\zeta}+\tilde{\xi}(t), y))
\end{aligned}
$$

However, we have, by denoting $\tilde{\lambda}_{+}$the exponent playing the same role for $\tilde{\phi}$ as the real number $\lambda_{+}$in (2.2.b): $g(\tilde{\phi}(\zeta+\tilde{\xi}(t), y)) \leq C e^{\tilde{\lambda}_{+} \tilde{\zeta}} \leq$ $C e^{-2 \tilde{\lambda}_{+} M} \cdot e^{\tilde{\lambda}_{+} \zeta} \cdot e^{-(c-\tilde{c}) t}$.

We therefore require $q$ to satisfy the inequality - notice that $\eta$, and therefore $\rho$ and $\tilde{\rho}$ may be chosen small enough to be $<\tilde{\lambda}_{+}-$:

$$
q^{\prime}(t)+\rho(c-\eta) q(t) \geq \frac{C}{\psi_{-}(y)} e^{-2 \tilde{\lambda}_{+} M} e^{-(c-\tilde{c}) t} .
$$

Case 2. $-A \leq \zeta \leq A$.

This time we still have $\tilde{\zeta} \leq-A$ and $\varphi(t, x, y)=w(\zeta-A, y)$. We get:

$$
\begin{aligned}
\mathcal{N} \underline{u}= & \xi^{\prime}(t) \phi_{x}(\zeta+\xi(t), y)+\tilde{\xi}^{\prime}(t) \tilde{\phi}_{x}(\tilde{\zeta}+\tilde{\xi}(t), y) \\
& -\left(q^{\prime}(t)+\rho(c-\eta) q\right) w(\zeta-A, y)+g(\tilde{\phi}(\tilde{\zeta}+\tilde{\xi}(t), y))-g(\underline{u})
\end{aligned}
$$

However there holds:

$$
\begin{aligned}
g(\tilde{\phi}(\zeta+\tilde{\xi}(t), y))-g(\underline{u}) & \leq\|g\|_{\text {Lip }}(1-\tilde{\phi}(\tilde{\zeta}+\tilde{\xi}(t), y)+q) \\
& \leq C\left(e^{\tilde{\lambda}_{+} \zeta} \cdot e^{-2 \tilde{\lambda}_{+} M} \cdot e^{-(c-\tilde{c}) t}+q\right)
\end{aligned}
$$

Set $m=\inf _{(x, y) \in \Sigma_{A}} \phi_{x}(x, y)$; it is sufficient to require:

$$
\xi^{\prime}(t) \leq \frac{q^{\prime}(t)}{m}-\frac{\|g\|_{L i p}}{m}\left(q(t)+C e^{-2 \tilde{\lambda}_{+} M} \cdot e^{-(c-\tilde{c}) t}\right) .
$$

Case 3. $\zeta \geq A$.

In this case three subcases have to be considered, namely: $\tilde{\zeta} \geq A$, $\tilde{\zeta} \in[-A, A], \tilde{\zeta} \leq-A$. The two first subcases lead to inequations similar 
to (8.5.a) and (8.5.b); the only differences is that they now involve $\tilde{\xi}$ and $\zeta$ instead of $\xi$ and $\tilde{\zeta}$. Therefore turn to the case $\tilde{\zeta} \leq-A$. In view of (8.3.a) and (2.2), there exists $\gamma>0, C>0$ such that there holds:

$$
\begin{aligned}
\mathcal{N} \underline{u} \leq & \xi^{\prime}(t) \phi_{x}(\zeta+\xi(t), y)+\tilde{\xi}^{\prime}(t) \tilde{\phi}_{x}(\tilde{\zeta}+\tilde{\xi}(t), y) \\
& -q^{\prime}(t) \varphi(t, x, y)+g(\phi(\zeta+\xi(t), y))+g(\tilde{\phi}(\tilde{\zeta}+\tilde{\xi}(t), y))-g(\underline{u}) \\
\leq & -\left(q^{\prime}(t)+\gamma q(t)\right) \varphi(t, x, y)+C \inf \left(e^{-\lambda \zeta}, e^{\lambda \tilde{\zeta}}\right)
\end{aligned}
$$

Here we have set $\lambda=\inf \left(\tilde{\lambda}_{+}, \lambda_{+}\right)$. The real number $\gamma$ is equal to $\frac{-g^{\prime}(1)}{2}$ $\left(\right.$ resp. $\left.\frac{-g^{\prime}(1)}{2}+\rho(c-\eta), \frac{-g^{\prime}(1)}{2}+\tilde{\rho}(\eta-\tilde{c})\right)$ if $\varphi(t, x, y)$ is equal to 1 (resp. $w(\zeta-A, y), \tilde{w}(\tilde{\zeta}-A, y)$.)

There holds: $\inf \left(e^{\lambda \zeta}, e^{\lambda \bar{\zeta}}\right)=O\left(e^{2 \lambda M} e^{-(c c \bar{c}) t}\right)$. Therefore we ask $q$ to satisfy a a similar inequality as (8.5.a).

To sum up, if we wish $\underline{u}(t, x, y)$ defined by (8.4) to be a subsolution, the functions $q, \xi$ and $\tilde{\xi}$ have to satisfy the following three additional conditions, for some $C>0, \lambda>0$ and $\gamma \in] 0, \lambda[$ :

$$
\begin{aligned}
& q^{\prime}(t)+\gamma q(t) \geq C e^{-2 \lambda M} \cdot e^{-(c-\check{c}) t}, \quad q(0)=\varepsilon, \\
& \xi^{\prime}(t), \tilde{\xi}^{\prime}(t) \leq C\left(q^{\prime}(t)-q(t)-e^{-2 \lambda M} \cdot e^{-(c-\tilde{c}) t}\right)
\end{aligned}
$$

Clearly, conditions (C1)-(C5) are fulfilled as soon as $\varepsilon$ is small enough and $M$ is large enough. Therefore, once they are chosen, $\underline{u}(t, x, y)$ defined by (8.4) is a subsolution.

5. The conclusion is now casy: if $\eta>0$ is chosen small enough in (8.2), then there exists $b>0$ such that $\underline{u}(0)<0$ on $\Sigma \backslash \Sigma_{b}$. Further, $\underline{u}(0)<1$, by construction. By Lemma 8.1 , there exists $t_{0}>0$ such that $\mathcal{S}\left(t_{0}\right) u_{0} \geq \underline{u}(0)$, and Lemma 8.2 follows from the maximum principle, with $r$ small enough.

\subsection{Proofs of the propagation theorems}

In this paragraph, we drop the assumption $\langle\alpha\rangle=0$. Let $\Gamma(x)$ be a nondecreasing, $C^{\infty}$ function, such that: $\Gamma(x)=0$ if $x \leq 0$ and $\Gamma(x)=1$ if $x \geq 1$. As in [11], we introduce the "left truncation" of $\mathcal{S}(t) u_{0}$ :

$$
v^{l}(t, x, y)=\left\{\begin{aligned}
\mathcal{S}(t) u_{0} & \text { if } x \leq \frac{c+\tilde{c}}{2} t \\
1-\Gamma(x)\left(1-\mathcal{S}(t) u_{0}\right) & \text { if } x \geq \frac{c+\tilde{c}}{2} t
\end{aligned}\right.
$$

Vol. 14, no 4-1997. 
The "right truncation" $v^{r}$ is defined in a symmetric way. We will show that $v^{l}$ converges exponentially to a travelling front; the same result can be proved in a similar way for $v^{r}$. This will imply our theorems.

We come back to the reference frame of $\phi$, still denoting by $x$ and $y$ the new coordinates. As in Section 4, we have the

COROLlary 8.3. - Select $\delta>0$. In both cases $A 1$ and A2, the set $\left\{v^{l}(t+),. t \geq \delta\right\}$ is relatively compact in $C_{l o c}([0,+\infty[, X)$.

Proof of Theorem 3.3 and 3.4, part 2. - Let $\omega\left(v_{0}^{l}\right)$ be the $\omega$-limit set of $v_{0}^{l}$; from Corollary 8.3 it is nonempty; therefore chose $u_{0} \in \omega\left(v_{0}^{l}\right)$. By Lemmas 8.1 and 8.2, there exist $h_{1} \leq h_{2}$ such that $\tau_{h_{1}} \phi \leq u_{0} \leq \tau_{l_{2} 2} \phi$. Therefore, by Theorem 3.1 (bistable case) or Theorem 3.2 (ignition temperature case) there exists $k \in \mathbb{R}$ such that $\mathcal{S}(t) u_{0}$ converges towards $\tau_{k} \phi$.

Consider now the real numbers $\delta_{0}$ and $\mu_{0}$ such that Corollary 2.2 works with $\tau_{k} \phi$, and select $\left.\delta \in\right] 0, \delta_{0}\left[\right.$. Denote the convergence exponent of $\mathcal{S}(t) u_{0}$ to $\tau_{k} \phi$ by $2 \omega$; for every $\left.\mu \in\right] 0, \mu_{0}[$, we may always assume - possibly by performing a sufficiently large translation in time - that $v^{l}$ satisfies an evolution equation of the type (2.8), with $\|h\|_{X^{2 \omega}} \leq \mu$. Select $T>0$ such that

$$
\left\|\mathcal{S}(T) u_{0}-\tau_{k} \phi\right\|_{X} \leq \frac{\delta}{2}
$$

from Corollary 8.3 there exists some large $t_{0}$ such that

$$
\left\|v^{l}\left(t_{0}+T\right)-\mathcal{S}(T) u_{0}\right\|_{X} \leq \frac{\delta}{2}
$$

Application of Corollary 2.2 shows that $v^{l}(t)$ converges to some translate of $\tau_{h} \phi$ of order $\delta+\mu$; since $\delta$ and $\mu$ are arbitrarily small, $v^{l}(t)$ converges to $\tau_{k} \phi$. The exponential convergence is also guaranteed by Corollary 2.2. •

It should be noticed that there is an intermediate behaviour between propagation of two flame fronts and extinction. To be convinced of this, one only has to notice that, due to the preceding results, propagation and extinction are both "open" properties, i.e. that if one of the two behaviours holds for an initial datum, it will hold for all nearby initial data. This is true because $\mathcal{S}(t) u_{0}$ has to be below $\frac{\theta}{2}$ in finite time for extinction, or to be above a nontrivial solution of (7.2). Therefore what really plays a role here is the continuous dependence with respect to the initial data. As a consequence, by connectedness, there has to be an in-between behaviour. 
Proof of Theorem 3.5. - It suffices to prove an inequality of the type (8.1), with $c=c_{*}$. The upper bound is easily obtained, due to the fact that $u_{0}$ is compactly supported.

The lower bound requires more care. Let $\underline{g}$ be a $C^{3}$ function such that

$$
\underline{g}(0)=0, \underline{g}^{\prime}(0)=g^{\prime}(0), \underline{g} \text { is concave. }
$$

There exists a unique $\theta_{1}>0$ such that $\underline{g}\left(\theta_{1}\right)=0$. From Theorem 3.2 of [22], we see that $\mathcal{S}(t) u_{0}$ becomes greater than $\theta_{1}$ on every compact subset of $\bar{\Sigma}$. Let us then consider a sequence $\left(g_{\theta}\right)_{\theta>0}$ such that, for all $\theta>0, g_{\theta}$ satisfies the assumptions of Case $\mathrm{A} 2$, and

$$
g_{\theta} \leq g \quad \lim _{\theta \rightarrow 0} g_{\theta}=g \text { in } \mathrm{C}([0,1]) .
$$

Let $c_{\theta}$ and $\tilde{c}_{\theta}$ the corresponding wave speeds; from [9] we know:

$$
\lim _{\theta \rightarrow 0} c_{\theta}=c_{*}, \quad \lim _{\theta \rightarrow 0} \tilde{c}_{\theta}=\tilde{c}_{*} .
$$

Applying Lemma 8.2 to every $g_{\theta}$ - it works uniformly for all $\theta^{\prime} \leq \frac{\theta_{1}}{2}$ then passing to the limit $\theta \rightarrow 0$ yields the desired inequality.

Proof of Theorem 3.6, part 2. - Once again, nothing more sophisticated than continuity of the nonlinear semigroup and parabolic regularity is involved in this result. Let $L>0, \delta>0$ and $v_{0} \in U C(\bar{\Sigma})$ be chosen so that

(i) $u_{0} \neq v_{0}$ only on $\Sigma_{L, \omega \backslash \omega^{\prime \prime}}$,

(ii) $v_{0}$ satisfies (3.4),

(iii) Theorem 3.4 holds with $L-1$ and $\frac{\eta}{2}$ instead of $L$ and $\eta$.

Choose $p>n$. By elementary $L^{p}$-continuity of $\mathcal{S}(t)$ - the reader who is still unfamiliar with the argument may consult [16] - we have, for every $t>0$ :

$$
\left\|\mathcal{S}(t) u_{0}-\mathcal{S}(t) v_{0}\right\|_{L^{p}(\Sigma)} \leq 2 C e^{C t}\left\|u_{0}-v_{0}\right\|_{L^{p}(\Sigma)} \leq L C e^{C t}\left|\omega \backslash \omega^{\prime \prime}\right| .
$$

By abstract regularizing effect (see [16], Chapter 3) we have, for possibly different $C$ :

$$
\left\|\mathcal{S}(1) u_{0}-\mathcal{S}(1) v_{0}\right\|_{L^{\infty}(\Sigma)} \leq L C\left|\omega \backslash \omega^{\prime \prime}\right| .
$$

Choosing $\left|\omega \backslash \omega^{\prime \prime}\right|$ small enough leads to the fact that, at time $t=1$, the solution $\mathcal{S}(t) u_{0}$ is above $\theta+\frac{\eta}{2}$ on $\Sigma_{L}$. In view of (iii), $\mathcal{S}(t) u_{0}$ develops into a pair of two fronts, and this terminates the proof of our long-time behaviour results. 


\section{DIRICHLET BOUNDARY CONDITIONS}

This is devoted to case B, and we come back to the arguments of Section 5. Since they have already been presented in detail, we will only insist on the technical differences. The section is divided into three paragraphs: in the first one, we state a uniform - possibly known - positivity result; in the second one, we prove Theorem 3.7. As in Section 5, we work in the reference frame of a travelling front.

\subsection{Refined Hopf Lemma}

We state the form that will be of use to us. On $Q=\mathbb{R}_{+} \times \Sigma$ consider a nonnegative strong solution of

$$
u_{t}-\Delta u+B(t, x, y) \cdot \nabla u+c(t, x, y) u=0 .
$$

with Dirichlet boundary conditions. The coefficients $B$ and $c$ belong to $C^{\delta, \frac{\gamma}{2}}(\bar{Q})$; moreover we assume

$$
\|B\|_{C^{\wedge} \cdot \frac{\hat{2}}{2}(Q)}+\|c\|_{C^{\wedge} \frac{\kappa}{2}(Q)} \leq \mu .
$$

From the usual Hopf Lemma [30] we shall prove

Proposition 9.1. - For every $M>0$, there exists $\mu(M)>0$ such that:

$$
\forall(t, x, y) \in\left[1,+\infty\left[\times \Sigma_{M}, \quad u(t, x, y) \geq \mu(M) d(y, \partial \omega) .\right.\right.
$$

Proof. - Assume the existence of a sequence $\left(t_{n}\right)_{n}$ going to $+\infty$, and of a sequence $\left(x_{n}, y_{n}\right)_{n} \in \Sigma_{M}$ such that

$$
\lim _{n \rightarrow+\infty} \frac{u\left(t_{n}, x_{n}, y_{n}\right)}{d\left(y_{n}, \partial \omega\right)}=0 .
$$

We may assume that $\left(x_{n}, y_{n}\right) \rightarrow\left(x_{\infty}, y_{\infty}\right) \in \bar{\Sigma}_{M}$. For $t \in[0,1]$, $(x, y) \in \bar{\Sigma}_{M}$ and $n$ large enough, let us set $u_{n}(l, x, y)=u\left(t_{n}-1+t, x, y\right)$. Due to the smoothness assumptions (9.2) and the classical Schauder estimates, the sequence $\left(u_{n}\right)_{n}$ converges, in $C^{2+\delta .1+\frac{\hat{t}}{2}}\left([0,1] \times \bar{\Sigma}_{A}\right)$, to a solution $u_{\infty}$ of an equation of the form

$$
u_{l}-\Delta u+B_{\infty}(t, x, y) . \nabla u_{\infty}+c_{\infty}(t, x, y) u_{\infty}=0,
$$

with Dirichlet boundary conditions. Two cases hold:

- Case 1. $y \in \omega$. Then (9.3) implies $u\left(1, x_{\infty}, y_{\infty}\right)=0$, a situation precluded by the strong maximum principle. 
- Case 2. $y \in \partial \omega$. Then (9.3) implies $u\left(1, x_{\infty}, y_{\infty}\right)=u_{\nu}\left(1, x_{\infty}, y_{\infty}\right)=$ 0 , a situation which is this tipme precluded by the Hopf boundary Lemma.

Therefore Proposition 9.1 is proved.

Remark. - This proposition could also have served us in the proof of Proposition 5.2. However, the Harnack inequalities could be readily used. Morenver, they need less smoothness than (9.2).

\subsection{Convergence for $x$-increasing initial data, eventual monotonicity and convergence}

We first prove the equivalent to Proposition 4.4 in [27]. We take the notations of Section 2, 2.3. In $X$, let $Y$ be the space of those functions $u$ which are $x$-increasing, and such that $\lim _{x \rightarrow-\infty} u(x, y)=\psi_{1}(y)$ and $\lim _{x \rightarrow+\infty} u(x, y)=\psi_{2}(y)$.

Proposition 9.2. - Let $u_{10}$ belong to $Y$. There exists $\varepsilon_{0}\left(u_{10}>0\right.$ and a constant $C$ such that, for every $\varepsilon \leq \varepsilon_{0}\left(u_{10}\right)$ and for every $u_{20} \in\left(u_{10}, \varepsilon\right)$, the following estimate holds:

$$
\left\|\mathcal{S}(t) u_{10}-\mathcal{S}(t) u_{20}\right\|_{X} \leq C \varepsilon .
$$

Proof. - In two steps. The new one is really the first.

1. Let $\gamma(x)$ be a standard regularisation of the distribution $\delta_{0}$, and $H$ the heaviside function. For $i \in\{1,2\}$ let $\phi_{i}$ be a positive eigenvector associated to $\mu_{1}\left(-\Delta_{y}-g_{y}\left(y, \psi_{i}\right)\right)$, and let us set

$$
\varphi(x, y)=\int_{-\infty}^{+\infty} \gamma\left(x-x^{\prime}\right)\left(H\left(-x^{\prime}\right) \phi_{1}(y)+H\left(x^{\prime}\right) \phi_{2}(y)\right) d x^{\prime}
$$

Obviously, there holds

$$
\begin{aligned}
& \left.\left.\varphi=\phi_{1} \text { on }\right]-\infty,-2\right] \times \omega \\
& \varphi=\phi_{2} \text { on }[2,+\infty[\times \omega
\end{aligned}
$$

and the following estimate is easily checked

$$
\left|-\Delta \varphi+(c+\alpha(y)) \varphi_{x}\right|+|\varphi| \leq C d(y, \partial \omega)
$$

2. A subsolution to (1.1) is looked under the form

$$
\underline{u}(t, x, y)=\mathcal{S}(t) u_{10}(\eta, y)-\varepsilon e^{-\lambda t} \varphi(\eta, y)
$$

with $\eta=x+\xi_{1}(t)$. For the choice of $\lambda, \xi_{1}(t)$ and $\varepsilon$, one basically proceeds as in [27], Section 4, by adding the following ingredients: for $\eta$ outside Vol. 14, $n^{\circ} 4-1997$. 
a large cylinder $\Sigma_{M}$, by reducing $\varepsilon$ a first time and taking (9.6.a) into account, one chooses

$$
\lambda \leq \frac{1}{2} \min \left\{\mu_{1}\left(-\Delta_{y}-g_{y}\left(y, \psi_{1}\right)\right), \mu_{1}\left(-\Delta_{y}-g_{y}\left(y, \psi_{2}\right)\right)\right\}
$$

Inside $\Sigma_{M}$, we have to choose, by virtue of estimate $(9.6 . \mathrm{b})$ :

$$
\xi_{1}^{\prime}(t)\left(u_{x}-\varepsilon \varphi_{x}\right) \leq O(\varepsilon) e^{-\lambda t} d(y, \partial \omega)
$$

Proposition 9.1 and estimate (9.6.b) again allow us to choose $\xi_{1}(t)=O(\varepsilon)$, which ends the proof of Proposition 9.2.

Proof of Theorem 3.7. - From Proposition 9.2, the set of initial data $u_{0}$ such that $\mathcal{S}(t) u_{0}$ converges to a front s open and closed in $Y$; moreover, due to local stability, it is nonempty. Therefore, it is $Y$ itself. Therefore it suffices to prove that, for $u_{0} \in X, \omega\left(u_{0}\right) \cap Y$ is nonempty. To do so we simply have to follow the scheme of the proof of Proposition 5.2, the only difference being that, this time, Proposition 9.1 instead of Proposition 5.1 applies.

\section{THE CASE OF THE SYSTEM}

This part is devoted to the proof of Theorem 3.8 on System (1.8). We immediately set ourselves in the reference frame of a wave, and we set, still keeping the notations $x$ and $y$ :

$$
W(t, x, y)=u(t, x, y)+v(t, x, y)-1
$$

We may write (1.8) under the form

(10.1.a) $u_{t}-\Delta u+(c+\alpha(y)) u_{x}=g(u)+f(u) W \quad$ for $(x, y) \in \Sigma$,

$$
\frac{\partial u}{\partial \nu}=0 \quad \text { for }(x, y) \in \mathbb{R} \times \partial \omega
$$

Therefore System (1.8) is a perturbation of (1.1). What prevents us from applying directly Corollary 2.3 , as we had done in the preceding sections, is that $W(t)$ does not decay exponentially as $t \rightarrow+\infty$. Therefore we have to use an additional result, which is a slight variant of a theorem of [14]. Here is the precise result. 
Let $X \subset \tilde{X}$ be two Banach spaces, with continuous injection from $X$ to $\tilde{X}$. Let $A$ be a sectorial operator in $X$ and $\tilde{X}$, and $f$ a $C^{2}$ function of $X$. Assume that the equation $A u=f(u)$ admits a $C^{2}$ manifold of solutions:

$$
\lambda \mapsto \phi_{\lambda}, \quad \lambda \in \mathbb{R} .
$$

We are interested in the stability of the $\phi_{\lambda}$ with respect to the evolution problem

$$
\frac{d u}{d t}+A u=f(u)+g(t) .
$$

The Cauchy Problem for (10.3), associated to an initial datum $u_{0}$, has a local solution denoted by $\mathcal{S}(t) u_{0}$. Let denote by $\mathcal{L}_{\lambda}$ the operator $A-f^{\prime}\left(\phi_{\lambda}\right)$. Its spectrum in $X$ (resp. $\tilde{X}$ ) will be denoted by $\sigma_{X}\left(\mathcal{L}_{\lambda}\right)$ (resp. $\left.\sigma_{\tilde{X}}\left(\mathcal{L}_{\lambda}\right)\right)$.

THEOREM 10.1. - Assume that the three following assumptions hold.

1. The function $f$ is $C^{2}$ in. X. Furthermore, the function $g$ satisfies the folloxing assumptions:

$$
\lim _{t \rightarrow+\infty}\|g(t)\|_{X}=0, \quad g \in \tilde{X}^{\omega} \text { for some } \omega>0 .
$$

For the meaning of the notation, see Notation 4 in Section 2.

2. For any given $\lambda \in \mathbb{R}, N\left(\mathcal{L}_{\lambda}\right)$ is spanned by $\phi_{\lambda}$; moreover the decompositions

$$
X=N\left(\mathcal{L}_{\lambda}\right) \oplus R_{\tilde{X}}\left(\mathcal{L}_{\lambda}\right), \quad \tilde{X}=N\left(\mathcal{L}_{\lambda}\right) \oplus R_{\tilde{X}}\left(\mathcal{L}_{\lambda}\right)
$$

hold, with algebraic and topological sum. Further, $\sigma_{\bar{X}}\left(\mathcal{L}_{\lambda}\right) \backslash\{0\}$ is contained in a cone of the complex plane with positive vertex and aperture $<\pi$.

3. For any given $\lambda$, let $e_{\lambda}^{*}$ be such that $\left\langle e_{\lambda}^{*}, \phi_{\lambda}{ }^{\prime}\right\rangle=1$ and $\mathcal{L}_{\lambda} e_{\lambda}^{*}=0$. There is a positive function $\varphi(t)$, such that $\lim _{t \rightarrow+\infty} \varphi(t)=0$, such that there holds, for all $v_{0} \in X$ :

$$
\left\|e^{-t \mathcal{L}_{\lambda}} v_{0}-<e_{\lambda}^{*}, v_{0}>\phi_{\lambda}{ }^{\prime}\right\|_{X} \leq\left\|v_{0}\right\|_{X} \varphi(t) .
$$

Let $\lambda_{0} \in \mathbb{R}$ and $v_{0} \in X$ be given. The Cauchy datum $u_{0}$ is taken under the form $u_{0}=\phi_{\lambda_{0}}+v_{0}, v_{0} \in X$. If $\left\|v_{0}\right\|_{X}$ and $\|g\|_{\tilde{X}^{-}}$are small enough, there exists $\lambda^{\infty}$ such that

$$
\lim _{t \rightarrow+\infty} \mathcal{S}(t) u_{0}=\phi_{\lambda_{\infty}} \quad \text { in } X .
$$

Vol. 14, $n^{\circ}$ 4-1997. 
When $X=\tilde{X}$, the above result is nothing else than Exercise 6 page 108 in [16], and the convergence of $\mathcal{S}(t) u_{0}$ to $\phi_{\lambda_{x}}$ is exponential. The proof of the general case will therefore closely follow the one in [16].

Proof. - When $v_{0}$ is small enough we may write, at least for short times: $u(t)=\phi_{\lambda(t)}+v(t)$, with $v(t) \in R\left(\mathcal{L}_{\lambda_{0}}\right)$. The problem is to show the validity of this formula for large times. Let $\Pi$ be the projection onto $R\left(\mathcal{L}_{\lambda_{1}}\right)$, and let us set

$$
\left.f(u+h)-f(u)=f^{\prime} u\right) h+Q(u, h), \quad\|Q(u, h)\|_{X} \leq C(u, h)\|h\|_{\mathrm{X}}^{2},
$$

where $C(u, h)$ is locally bounded. Fquation (10.3) may be written under the form

$$
\begin{aligned}
\dot{\lambda}(t) & =\frac{\left\langle e_{\lambda_{0}}^{*}, Q(\lambda(t), v(t)) v(t)+R(t, v(t)+g(t)\rangle\right.}{\left\langle e_{\lambda_{0}}^{*}, \phi_{\lambda}^{\prime}(t)\right\rangle} \\
\dot{v}+\mathcal{L}_{\lambda_{0}} v & =\Pi Q(\lambda, v)-\dot{\lambda}(t) \Pi \phi_{\lambda(t)}^{\prime}+\Pi R(t, v)+\Pi g(t)
\end{aligned}
$$

with

$$
R(t, u)=\int_{0}^{1} f^{\prime \prime}\left(\phi_{\lambda(t)}+\sigma \phi_{\lambda_{0}}\right) \cdot\left(\phi_{\lambda(t)}-\phi_{\lambda_{0}}\right) \cdot u d \sigma
$$

Let us assume, as is usual: $\left|\lambda(t)-\lambda_{0}\right|+\|v(t)\|_{z} \leq \eta$. We operate in $\tilde{X}$; writing down the integral formula for $v(t)$, using the formula for $\dot{\lambda}(t)$ and carrying on with the usual estimtes yields the existence of $\omega>0$ and two constants $C_{1}$ and $C_{2}(\eta)$, bounded for small $\eta$, such that

$$
\begin{aligned}
& \|v(t)\|_{\tilde{X}} \\
& \leq C_{1} e^{-\omega t}\left\|v_{0}\right\|_{X}+C_{2}(\eta) \int_{0}^{t} e^{-\omega(t-s)}\left(\|v(s)\|_{X}\right. \\
& \left.\quad+\|g(s)\|_{\tilde{X}}+\left|\lambda(s)-\lambda_{0}\right|\right)\|v\|_{\tilde{X}} d s \\
& \quad \leq\left(C_{1}+C_{2}(\eta)\right)\left(e^{-\omega t}\left(\left\|v_{0}\right\|_{\mathrm{X}}+\|g\|_{\tilde{X}^{-} \omega}\right)+\eta \int_{0}^{t} e^{-\omega(t-s)}\|v\|_{\tilde{X}} d s\right) \\
& \quad \leq 2\left(C_{1}+C_{2}(\eta)\right)\left(\left\|v_{0}\right\|_{Z}+\|g\|_{\tilde{X}^{-} \omega}\right) e^{-\left(\omega-2\left(C_{1}+C_{2}(\eta)\right) \eta\right) t}
\end{aligned}
$$

Looking at the equation for $\lambda(t)$ in (10.4) we infer the existence of a constant $q_{1}>0$ and a function $q_{2}(\eta)$, with $\lim _{\eta \rightarrow 0} q_{2}(\eta)=0$ such that

$$
|\dot{\lambda}(t)| \leq \frac{q_{1}}{1-q_{2}(\eta)}\left(\|v\|_{Z}+\left|\lambda-\lambda_{0}\right|\right)\|v\|_{\tilde{X}}
$$


Therefore, when $\eta$ and $\|g\|_{\tilde{x}_{\omega}}$ are really small enough, we have $\left|\lambda-\lambda_{0}\right| \leq \eta$, and there exists $C_{3}(\eta)$ such that

$$
\left|\lambda(t)-\lambda_{0}\right|+\|v(t)\|_{\hat{Y}} \leq C_{3}(\eta) \eta e^{-\frac{\omega}{2} t} .
$$

It now remains to prove that $\|v(t)\|_{X}$ actually remains below $\eta$. The above formulas will from then on be valid, and the theorem will be proved. We write the integral formula for $v$ in $Z$, and we use (10.5); this yields

$$
\|v(t)\|_{x} \leq C\left\|v_{0}\right\|_{x}\left(\varphi(t)+\eta \int_{0}^{t} \varphi(t-s) e^{-\frac{\omega}{2} s} d s\right) .
$$

Therefore, when $\left\|v_{0}\right\|_{X}$ is small enough, we have $\|v(t)\|_{Z} \leq \eta$.

Theorem 10.1 will provide us for an equivalent version of the compactness theorems in Section 4.

LeMmA 10.2. - Let $u_{0}$ and $v_{0}$ be as in Theorem 3.8. For $t_{0}>0$ large enough there exist $q>0, \omega>0$, and $\varphi(t)>0$, such that $\lim _{t \rightarrow+\infty} \varphi(t)=0$, and such that:

$$
\left.\forall t \geq t_{0}, \quad \tau_{\xi_{1}} \phi-\varphi(t)\right) \leq u(t, x, y) \leq \tau_{\xi_{2}} \phi+\varphi(t)
$$

Proof. - We remark that the function $W(t)$ satisfies $\lim _{t \rightarrow+\infty}\|W(t)\|_{\infty}=0$; moreover, for every $\varepsilon>0$, there exists $t_{\varepsilon}>0$ such that

$$
-\varepsilon e^{-\omega t} \leq e^{-r x} W(t) \leq \varepsilon e^{-\omega t} \quad \text { for } t \geq t_{\tilde{\varepsilon}} .
$$

Therefore we only have to apply Theorem 10.1 with

$$
\tilde{X}=\left\{u: e^{-r x} u \in U C(\bar{\Sigma})\right\},
$$

and the following choice of nonautonomous terms: $g_{1}(t)=-\varepsilon e^{-\omega t}$ for the lower bound, and $g_{2}(t)=\varepsilon e^{-\omega t}$ for the upper bound.

Proof of Theorem 3.8. - Same as the proofs of Theorems 3.3 and 3.4, Part 2.

\section{EXTENSIONS AND CONCLUDING REMARKS}

The method that we have developped in this paper for proving eventual monotonicity, combined to the techniques that we have used in our previous papers [7], [22], [26], [27], also allows us to handle more general differential Vol. 14, $n^{\circ}$ 4-1997. 
operators, namely to replace the term $-\Delta u$ in (1.1) by an expression of the type $-\left(\sum_{1 \leq i, j \leq N} a_{i j}(y, u) \partial_{x_{i}, x_{j}}^{2} u\right)$, or also of the type $-(\operatorname{div}(D(y, u) \nabla u))$. In particular, for $N=1$, one can prove in a similar way as above that the travelling front solutions of the problem

$$
u_{t}-\left(D(u) u_{x}\right)_{x}=g(u)
$$

$g$ being as in case A1-A3/ZFK or B, are globally stable. Local stability has been known for a long time (it can easily be deduced from [29]), but global stability, up to our knowledge, is new.

In the same vein, we can also prove convergence to travelling waves for the solutions viscous regularisations of hyperbolic equations, namely equations of the form

$$
\begin{aligned}
u_{t}-\mu u_{x x}+(f(u))_{x} & =g(u) \quad x \in \mathbb{R} \\
u(t,-\infty)=1, u(t,+\infty) & =0 .
\end{aligned}
$$

The term $g$ may satisfy $\mathrm{Al}$, and the term $f$ is taken to be strictly convex. There exists an $x$-decreasing wave $\phi$ connecting 0 to 1 , which is a regularisation of the entropic shock wave $H(x-\sigma t)$, with $\sigma=f(1)-f(0)$; see [30] for the background. The stability of $\phi$ is once again well-known; see [16] or [22]; we provide here a method for proving its global stability, and the result is once again new.

In bounded domains, a similar study could be made in order to treat a problem of the form

$$
u_{\iota}=F\left(x, u, D u, D^{2} u\right)
$$

see Berestycki and Nirenberg [10] for the right assumptions of $F$ and the appropriate boundary conditions. Once sufficiently stong a priori estimates are known, a solution of the above problem becomes monotone in finite time in the whole domain. Such a result, however, has little interest, for the theory of Hirsch, combined to [10], asserts that every solution converges towards the unique monotone equilibrium state.

Let us now come back for a moment to Problem (7.1). The whole structure of the solution set is still to be understood. In particular, we have not yet fully exploited the fact that we were working in a continuous family of cylinders, which should yield a much more precise description of this solution set.

In the same way, we have said nearly nothing about the intermediate behaviour that we have noticed in Section 7.3. In one space dimension, at 
least for even initial data, one could effortlessly be a little more explicit. However, the full study of this critical behaviour would have led us too far from the main stream of this paper, and will be performed somewhere else.

Let us finally conclude with a question that we leave open, and whose answer might require other techniques than the ones we have displayed. In [22], where case A3 is investigated, we prove that the long-time behaviour of $\mathcal{S}(t) u_{0}$, strongly depends on the asymptotic behaviour at $x=-\infty$; namely the decay rate selects the speed of the front; we are even able to give an explicit expression of the asymptotic shift.

Here nothing like this happens; in fact, our convergence proof, which relies on a connectedness argument, is perfectly non-constructive. The problem occurs in one space dimension as well, for the Liapunov function which naturally comes up only tells that quasiconvergence to some equilibrium occurs, but never specifies which one is chosen, and why. Most certainly, it does not occur as in the KPP case, and here is a very simple reason why. For $u_{0} \in X$, let $h\left(u_{0}\right)$ be such that $\mathcal{S}(t) u_{0}$ converges to $\tau_{h\left(u_{0}\right)} \phi$.

Proposition 11.1. - For every $r, L>0$, for every function $a(y) \in$ $C\left(\bar{\omega}, \mathbb{R}_{+}\right)$, there exists $\left(u_{L}, v_{L}\right) \in X^{2}$ such that

(i) $u_{L}=v_{L}$ on $\left.]-\infty,-L\right] \times \omega$,

(ii) $\lim _{x \rightarrow-\infty} u_{L}(x, y) e^{-r \cdot x}=a(y)$ as $x \rightarrow-\infty$, uniformly in $y \in \omega$ and such that $h\left(u_{L}\right)-h\left(v_{L}\right)>L$.

Proof. - This is nothing else than an application of Theorem 2.1 to two suitably chosen initial data. We use the notations of Section 2 and Theorem 2.1.

Let $u_{L}$ and $v_{L}$ be defined as follows:

$$
\begin{gathered}
u_{L}(x, y)=\left\{\begin{array}{c}
\left.\left.a(y) e^{r x} \text { in }\right]-\infty,-L\right] \times \omega \\
\text { linear in } x \text { in }[-L,-L+1] \\
\phi(x, y) \text { in }[-L+1,+\infty[\times \omega
\end{array}\right. \\
v_{L}(x, y)=\left\{\begin{array}{c}
\left.\left.a(y) e^{r x} \text { in }\right]-\infty,-L\right] \times \omega \\
\text { linear in } x \text { in }[-L,-L+1] \\
\tau_{-2 L} \phi(x, y) \text { in }[-L+1,+\infty[\times \omega
\end{array}\right.
\end{gathered}
$$

When $L>0$ is large enough, the quantities $\left\|u_{L}-\phi\right\|_{X}$ and $\left\|\tau v_{2 L}-\phi\right\|_{X}$ become $<\delta_{0}$, and Theorem 2.1 applies, which yields:

$$
h\left(u_{L}\right)-h\left(\tau_{2 L} v_{L}\right)=\gamma\left(u_{L}\right)-\gamma\left(\tau_{2 L} v_{L}\right) ;
$$

Vol. $14, n^{\circ} 4-1997$. 
in other words:

$$
\begin{aligned}
h\left(u_{L}\right)-h\left(v_{L}\right) & =2 L+\gamma\left(u_{L}\right)-\gamma\left(\tau_{2 L} v_{L}\right) \\
& \geq 2 L-2 \sup _{v \in \mathcal{B} \backslash\left(0, \delta_{0}\right)}|\gamma(v)|
\end{aligned}
$$

This proves Proposition 11.1

Thus we see that convergence occurs in a totally different manner than in the KPP case: indeed, for $r>0$ small enough, assumptions (i) and (ii) of Proposition 9.1 would imply $h\left(u_{L}\right)=h\left(v_{L}\right)$.

Therefore we think that understanding how the asymptotic shift is selected in the bistable case and in the ignition temperature case would undoubtedly be mathematically quite interesting.

\section{APPENDIX}

This appendix contains an outline of the proof of Theorem 2.1 in the bistable case, of the proof of Corollary 2.2 in both cases. To be complete, we should also include the Dirichlet case: however it is quite similar to the proof that we are going to give now. First, turn to Theorem 2.1.

In the framework of the bistable case, recall that $X=U C(\bar{\Sigma})$. Let $L$ be the linearised operator about $\phi$, with domain

$$
D(L)=\left\{u \in X, \Delta u \in X, \quad \frac{\partial u}{\partial \nu}=0 \text { on } \mathbb{R} \times \partial \omega\right\} .
$$

and whose expression is given by

$$
L u=-\Delta u+\beta(y) u_{x}-g^{\prime}(\phi) u .
$$

For $\lambda \in \mathbb{C}$, a solution of $L u=\lambda u$ is an element $u \neq 0$ of $D(L)$ such that $L u=\lambda u$. Two steps are required for Theorem 2.1, each of them being the subject of one paper in the ignition temperature case.

\section{Step 1. Linear stability}

It suffices to copy word by word the proofs of [7], so we will not dwell on this point any longer.

\section{Step 2. Nonlinear stability}

This part corresponds to [26]. There are once again two steps: first, one has to show that $\sigma(L) \backslash\{0\}$ lies in a cone of the complex plane, with angle 
$<\frac{\pi}{2}$, and with vertex on the positive real axis. Second, one has to show that 0 is a simple - in the geometric as well as in the algebraic sense - of $L$. The conclusion of the proof of Theorem 2.1 relies on an Implicit Functions Theorem argument due to Sattinger [29].

1. Localisation of the spectrum. - For $a>0$ and $\alpha \in] 0, \frac{\pi}{2}\left[\operatorname{let} C_{a, \alpha}\right.$ denote the cone of the complex plane

$$
C_{a, \alpha}=\{\lambda \in \mathbb{C}:|\arg (\lambda-a)|<\alpha\} .
$$

Proposition A.1. - The operator $L$ is sectorial in X. Further, there exist $a>0$ and $\alpha \in] 0, \frac{\pi}{2}\left[\right.$ such that $\sigma(L) \backslash\{0\} \subset C_{a, \alpha}$. Moreover, for every $\delta \in] 0, a\left[\right.$ and $\lambda \notin\left(C_{a, \alpha} \cup B_{\delta}\right)$, there holds:

$$
\left\|(\lambda I-L)^{-1}\right\|_{\mathcal{L}(U C(\bar{\Sigma}))} \leq \frac{C(\delta)}{|\lambda-a|} .
$$

Proof. - The fact that $L$ is sectorial is an extension of a result of Stewart [31]; see [26]. As for the existence of $C_{a, \alpha}$ we construct two operators $T$ and $S$ of $X$, such that $L=S+T$, Re $\sigma(L)>\gamma$ for some $\gamma>0$, and $S T^{-1}$ is compact. A result of Gohberg [15], combined to the fact that $L$ is sectorial, yields the existence of $C_{a, \alpha}$. Estimation (A.3) follows easily.

As for the operators $S$ and $T$, let $\Gamma(x)$ be a $C^{\infty}$ nondecreasing function, zero for $x \leq 0$, equal to 1 pour $x \geq 1$. We set:

$$
q(x)=g^{\prime}(0) \Gamma(x)+g^{\prime}(1)(1-\Gamma(x)) .
$$

There exists $\gamma>0$ such that $-q(x)>\gamma$.

We set $D(T)=D(L)$, and, for $u \in D(T)$ :

$$
T u=-\Delta u+\beta(y) u_{x}-q(x) u \text {; }
$$

then, for $u \in X, S u=\left[q(x)-g^{\prime}(\phi)\right] u$. We notice that $S$ and $T$ satisfy all the required properties (easy adaptation of [26], Section 4, Lemmas 4.6 and 4.7$)$.

2. multiplicity of the eigenvalue 0 . - In fact, we only have to prove that 0 is geometrically simple; the algebraic simplicity being obtained by copying the proof of Theorem 3.1 in [26].

Proposition A.2. - Let $u \in D(L)$ be such that $L u=0$. Then $u$ has the form Constant $\times \phi_{x}$. 
Proof. - We may assume that $u$ is real-valued. By the same argument as in Proposition 3.4 of [26], there exist $\alpha_{+}$and $\alpha_{-}$such that

$$
\lim _{x \rightarrow-\infty} \frac{u(x, y)}{\phi_{x}(x, y)}=\alpha_{-} . \quad \lim _{x \rightarrow+\infty} \frac{u(x, y)}{\phi_{x}(x, y)}=\alpha_{+} .
$$

We have to distinguish the case $\alpha_{-}=\alpha_{+}$and the case $\alpha_{-} \neq \alpha_{+}$. If $\alpha_{-}=\alpha_{+}$, the same argument as in Proposition 3.4 of [26] proves $u=\alpha_{+} \phi_{x}$. Therefore it remains to prove that $\alpha_{-} \neq \alpha_{+}$is impossible.

In order to do so, we always may assume that $\alpha_{-}=0$, and $\alpha_{+}>0$. On the other hand we know - see [9] - that there exist $\lambda_{-}>0$ and $\phi_{-}(y)>0$ such that:

$$
\phi_{x}(x, y)=e^{\lambda_{-} x} \phi_{-}(y)+O\left(e^{\left(\lambda_{-}+\varepsilon\right) x}\right)
$$

as $|x| \rightarrow-\infty$, for some $\varepsilon>0$. The maximum principle applied to $\frac{u}{\phi_{x}}$ yields: $u>0$ in $\bar{\Sigma}$. Let us prove by induction the following claim: for every $n \in \mathbb{N}, u(x, y)=O\left(e^{\left(n \lambda_{-}-\varepsilon_{n}\right) x}\right)$ as $|x| \rightarrow-\infty$, with $0<\varepsilon_{n}<\frac{\lambda_{-}}{2}$. This is indeed true for $n=1$ and $\varepsilon_{1}=\frac{\lambda}{3}$; therefore assume it is true up to the order $n$, and let us prove that it is true up to the order $n+1$. The equation $L u=0$ may be written as follows:

$$
L_{0} u:=-\Delta u+\beta(y) u_{x}-g^{\prime}(0) u=\left(g^{\prime}(\phi)-g^{\prime}(0)\right) u
$$

From Lemma 5.2 of [25] and the induction assumption, there exist $u^{0} \in D(L)$ solution of $L_{0} u^{0}=0$ and $u_{*} \in D(L)$ solution of

$$
L_{0} u_{*}=\left(g^{\prime}(\phi)-g^{\prime}(0)\right) u
$$

such that $u=u^{0}+u_{*}$, and:

$$
u_{*}(x, y)=O\left(e^{\left((n+1) \lambda_{-}-\varepsilon_{n}-\frac{\lambda_{-}}{3^{n+1}}\right) x}\right) .
$$

From [9], there exist $\gamma_{-} \in \mathbb{R}, \lambda_{n+1}>\lambda_{-}$and $\phi_{n+1}(y)$ such that:

- the function $u^{n+1}:=e^{\lambda_{n+1} x} \phi_{n+1}(y)$ belongs to $D(L)$, and satisfies $L_{0} u^{n+1}=0$; moreover the function $\phi_{n+1}$ is nonzero and changes sign in $\bar{\omega}$,

- the function $u^{0}$ may be written as:

$$
u^{0}=\gamma_{-} e^{\lambda_{-} x} \phi_{-}(y)+u^{n+1}(x, y)+o\left(e^{\lambda_{n+1} x}\right) .
$$


Since $\alpha_{-}=0$, then $\gamma_{-}=0$. Since the function $\phi_{n+1}$ changes sign in $\bar{\omega}$ and since $u>0$ in $\bar{\Sigma}$, we get:

$$
\lambda_{n+1} \geq(n+1) \lambda_{-}-\varepsilon_{n}-\frac{\lambda_{-}}{3^{n+1}} .
$$

Finally, setting $\varepsilon_{n+1}=\varepsilon_{n}+\frac{\lambda_{-}}{3^{n+1}}$, we see that: $0<\varepsilon_{n+1}<\frac{\lambda_{-}}{2}$. This proves our claim, since $u=u^{0}+u_{*}$.

Therefore, $u$ decays faster than any exponential as $x \rightarrow-\infty$. However, for $\Lambda>0$ large enough, the function $\underline{u}(x)=e^{\Lambda x}$ satisfies: $L \underline{u} \leq 0$ in $\bar{\Sigma}$. On the other hand, let $x_{0}<0$ be such that $g^{\prime}(\phi) \leq 0$ in ]$\left.-\infty, x_{0}\right] \times \omega$. Since $u>0$ in $\bar{\Sigma}$, there exists $\delta>0$ small enough so that: $u\left(x_{0},.\right) \geq \delta \underline{u}\left(x_{0},.\right)$, which implies, by the maximum principle: $u(x, y) \geq \delta \underline{u}(x, y)$ for $\left.(x, y) \in]-\infty, x_{0}\right] \times \omega$. Contradiction.

Remark. - When dealing with Dirichlet conditions, one should avoid divisions by $\phi_{x}$, and rather work with solutions of the form $u+k \phi_{x}$, for suitably chosen $k$.

Once we know what we have to know, the end of the proof of Theorem 2.1 is a particular case of the proof of Corollary 2.2 , with $h(t)=0$. This is what we are going to see now.

Proof of Theorem 2.1. and Corollary 2.2. - We transform Problem (2.8) into the following problem: find $v(t) \in X^{\omega}$ and $\gamma \in \mathbb{R}$ such that

$$
\begin{aligned}
\frac{d v}{d t}+L v & =R(h, v, \gamma) \\
v(0) & =v_{0}-\gamma \phi_{x}-k(\gamma)
\end{aligned}
$$

the functions $R$ an $k$ being the second-order terms:

$$
\begin{aligned}
R(h, v, \gamma) & =g\left(\tau_{\gamma} \phi+v\right)-g\left(\tau_{\gamma} \phi\right)-\left(g^{\prime}\left(\tau_{\gamma} \phi\right)-g^{\prime}(\phi)\right) v+h \\
k(\gamma) & =\tau_{\gamma} \phi-\phi-\gamma \phi_{x}
\end{aligned}
$$

We set: $v(t)=p(t) \phi_{x}+\zeta(t)$, with $\zeta(t) \in R(L)$; see [26], Section 5 to see why we may do so. Let $e^{*} \in X^{*}$ (the dual space of $X$ ) be such that $L^{*} e^{*}=0,\left\|e^{*}\right\|=1$ and $\left\langle e^{*}, \phi_{x}\right\rangle=1$. The equations for $p(t)$ and $\zeta(t)$ read:

$$
\begin{gathered}
\frac{d \zeta}{d t}+\mathcal{Q} L \zeta=\mathcal{Q} R(h, v, \gamma) \\
\frac{d p}{d t}=<e^{*}, R(h, v)> \\
\zeta(0)=\mathcal{Q}\left(v_{0}-k(\gamma)\right), \quad p(0)=<e^{*}, v_{0}>-\gamma-<e^{*}, k(\gamma)>
\end{gathered}
$$


Here, $\mathcal{Q}=\operatorname{Id}_{X}-\left\langle e^{*}, .>\phi_{x}\right.$; we know that there exists $\omega>0$ such that $\left\|e^{-t \mathscr{Q} L}\right\|_{X} \leq e^{-2 \omega t}$.

Problem (A.9) is equivalent to the following problem: find $\zeta(t) \in \mathcal{Q} X^{\omega}$, $p(t) \in E^{\omega}$ and $\gamma \in \mathbb{R}$ such that:

$$
\begin{array}{r}
\zeta(t)-e^{-t \mathcal{Q} L}\left(v_{0}-k(\gamma)\right)-\int_{0}^{t} e^{-(t-s) \mathcal{Q} L} R(h, v(s), \gamma) d s=0 \\
p(t)+\int_{t}^{+\infty}<e^{*}, R(h, v(s), \gamma)>d s=0 \\
\gamma-\left\langle e^{*}, v_{0}\right\rangle+<e^{*}, k(\gamma)>-\int_{0}^{+\infty}<e^{*}, R(h, v(s), \gamma)>d s=0
\end{array}
$$

We write (A.10) in compact form:

$$
\mathcal{F}_{1}\left(\zeta, p, \gamma, v_{0}, h\right)=\mathcal{F}_{2}\left(\zeta, p, \gamma, v_{0}, h\right)=\mathcal{F}_{3}\left(\zeta, p, \gamma, v_{0}, h\right)=0
$$

and set: $\mathcal{F}=\left(\mathcal{F}_{1}, \mathcal{F}_{2}, \mathcal{F}_{3}\right)$. The mapping $\mathcal{F}$ is $C^{1}$ from $\mathcal{Q} X^{\omega} \times E^{\omega} \times \mathbb{R} \times$ $X \times X^{2 \omega}$ to $\mathcal{Q} X^{\omega} \times E^{\omega} \times \mathbb{R}$; moreover $\mathcal{F}(0)=0$. Further, as easily seen we have $\partial_{\zeta, p, \gamma} \mathcal{F}(0)=\operatorname{Id}_{\mathcal{Q} X^{-}} \otimes \operatorname{Id}_{E^{\omega}} \otimes \mathrm{Id}_{\mathbb{R}}$, which clearly is an isomorphism of $\mathcal{Q} X^{\omega} \times E^{\omega} \times \mathbb{R}$. Application of the Implicit Function Theorem ends the proof of Corollary 2.2 .

\section{ACKNOWLEDGEMENTS}

This paper is the conclusion of a work initiated in my $\mathrm{PhD}$ thesis. It is my pleasure to express here my deep gratitude to my advisors Profs. H. Berestycki and B. Larrouturou for their patient guidance, helpful advice and constant encouragement.

I would also like to thank Prof. H. Matano for very stimulating discussions on the global stability problem, and two anonymous referees, whose relevant remarks substantially improved this paper.

\section{REFERENCES}

[1] S. Agmon and L. Nirenberg, Properties of solutions of ordinary differential equations in Banach space, Comm. Pure Appl. Math., Vol. 16, 1963, pp. 121-239.

[2] D. G. Aronson and H. F. Weinberger, Nonlinear diffusion in population genetics, combustion and nerve propagation, Partial differential equations and related topics, Lect. Notes in Math., Vol. 446, Springer Verlag, New-York, 1975, pp. 5-49. 
[3] D. G. Aronson and H. F. Weingerger, Multidimensional diffusion arising in population genetics, Adv. Math., Vol. 30, 1978, pp. 33-58.

[4] H. Berestycki, L. A. Caffarelli and L. Nirenberg, Uniform estimates for regularisations of free boundary problems, Analysis and partial differential equations, C. Sadosky \& M. Decker eds., 1990, pp. 567-617.

[5] H. BERESTYCKI and B. LARROUTUROU, Planar travelling front solutions of reaction-diffusion problems, to appear.

[6] H. Berestycki, B. Larrouturou and P. L. Lions, Multidimensional travelling wave solutions of a flame propagation model, Arch. Rat. Mech. Anal., Vol. 111, 1990, pp. 33-49.

[7] H. Berestycki, B. Larrouturou and J.M. RoQuejoffre, Stability of travelling fronts in a model for flame propagation, Part I: Linear analysis, Arch. Rat. Mech. Anal., Vol. 117, 1992, pp. 97-117.

[8] H. Berestycki and L. Nirenberg, Some qualitative properties of solutions of semilinear equations in cylindrical domains, Analysis et Cetera (dedicated to J. Moser), P. H. Rabinowitz \& E. Zehnder eds., Academic Press, New-York, 1990, pp. 115-164.

[9] H. Berestycki and L. Nirenberg, Travelling fronts in cylinders, Ann. IHP. Analyse non linéaire, Vol. 9, 1992, pp. 497-573.

[10] H. BERESTYCKI and L. NIRENBERG, On the method of moving planes and the sliding method, Bol. da So. Brasileira de Matematica, Vol. 22, 1991, pp. 1-37

[11] P. C. FIFE and J. B. McLEOD, The approach of solutions of nonlinear diffusion equations by travelling front solutions, Arch. Rat. Mech. Anal., Vol. 65, 1977, pp. 335-361.

[12] A. Friedman, Partial differential equations of parabolic type, Prentice Hall, 1964.

[13] B. Gidas, W. M. Ni and L. NirenberG, Symmetry and related properties via the maximum principle, Comm. Math. Phys., Vol. 68, 1979, pp. 209-243.

[14] L. Gl.angetas and J. M. RoQuejoffre, Bifurcations of travelling waves in the thermodiffusive model for flame propagation, Arch. Rat. Mech. Anal., Vol. 134, 1996. pp. $341-402$.

[15] I. C. GohberG and M. G. KREIN, Introduction to the theory of linear nonselfadjoint operators, Transl. Math. Monog. Vol. 18, Am. Math. Soc., Providence, R.I., 1969.

[16] D. Henry, Geometric theory of semilinear parabolic equations, Lecture Notes in Mathematics, Springer Verlag, New-York, 1981.

[17] M. W. HiRsch, Stability and convergence in strongly monotone dynamical systems, $J$. Reine Angew. Math., Vol. 383, 1988, pp. 1-53.

[18] A. V. IVANOV, The Harnack inequality for weak solutions of quasilinear parabolic equations of second order, Soviet Math. Dokl., Vol. 8, 1967, pp. 463-466.

[19] C. K. R. T. JonEs, Asymptotic behaviour of a reaction-diffusion equation in higher dimensions, Rocky Mountain J. Math., Vol. 13, 1983, pp. 355-364.

[20] O. A. LADYZHENSKAYA, V. A. SOLONNIKOV and N. N. URALCEVA, Linear and quasilinear equations of parabolic type, Transl. Math. Monog., Vol. 23, Am. Math. Soc., Providence. R.I, 1968.

[21] P. L. Lions, Structure of the set of steady-state solutions and asymptotic behaviour of semilinear heat equations, J. Diff. Eq., Vol. 53, 1984, pp. 362-386.

[22] J. F. MaLlordy and J. M. Roquejoffre, A parabolic equation of KPP type, SIAM J. Math. Anal., Vol. 26, 1995, pp. 1-20.

[23] H. MATANO, Strong comparison principles in nonlinear parabolic equations, Nonlinear parabolic equations: Qualitative properties of solutions, L. Boccardo \& A. Tesei eds., Pitman Longman 1987.

[24] H. Matano, Existence of nontrivial unstable sets for equilibriums in strongly orderpreserving dynamical systems, J. Fac. Sci. Univ. Tokyo, Vol. 30, 1983, pp. 645-673.

[25] A. PAZY, Asymptotic expansions of solutions of ordinary differential equations in Hilbert space, Arch. Rat. Mech. Anal, Vol. 24 1967, pp. 193-218.

[26] J. M. RoQueJofFRE, Stability of travelling fronts in a model for flame propagation, Part II: Nonlinear stability, Arch. Rat. Mech. Anal., Vol. 117, 1992, pp. 119-153.

Vol. 14, $n^{\circ} 4-1997$. 
[27] J. M. RoQueJoffRe, Convergence to travelling waves for solutions of a class of semilinear parabolic equations, J. Diff. Eq., Vol. 108, 1994, pp. 262-295.

[28] J. M. RoQueJofFrE and D. TERMAN, On the stability of steady planar premixed flames, Nonlinear analysis, TMA, Vol. 22, 1994, pp. 137-154.

[29] D. H. SatTinger, Stability of waves of nonlinear parabolic systems, Adv. Math., Vol. 22, 1976, pp. 312-355.

[30] J. SMOLLER, Shock waves and reaction diffusion equations, Grund. math. Wiss., Vol. 258, SPRINGER Verlag.

[31] H. B. STEWART, Generation of analytic semigroups by strongly elliptic operators under general boundary conditions, Trans. Am. Math. Soc., Vol. 259, 1980, pp. 299-310.

[32] J. M. VEGA, Multidimensional travelling wave fronts in a model from combustion theory and in related problems, Differential and Integral equations, Vol. 6 1993, pp. 131-155.

[33] J. B. Zeldovich and D. A. FRANK-KamenETSKiI, A theory of thermal propagation of flame, Acta Physiochimica URSS, Vol. 9, 1938.

(Manuscript received October 12, 1995; revised version received June $20,1996$. 